\title{
CLASIFICACIÓN DE SUELOS DE 15 ESTACIONES ACELEROGRÁFICAS, MEDIANTE EL USO DE MÉTODOS BASADOS EN VIBRACIONES AMBIENTALES Y DEL PARÁMETRO VS30
}

\author{
SOIL CLASSIFICATION IN 15 ACCELEROGRAPHIC STATIONS USING \\ METHODS BASED ON AMBIENT VIBRATIONS AND VS30
}

\author{
Víctor Schmidt \\ Laboratorio de Ingeniería Sísmica. Nivel 3-A Facultad de Ingeniería, \\ Universidad de Costa Rica, 2060, San Pedro de Montes de Oca. \\ victor.schmidt@ucr.ac.cr
}

(Recibido: 14/05/2014; aceptado: 28/06/2014)

\begin{abstract}
The H/V, FK and SPAC techniques were applied, all based on the measurement of ambient vibrations generated by surface waves for the characterization of soils beneath 15 selected sites, 12 of them corresponding to accelerographic stations of the Earthquake Engineering Laboratory of the University of Costa Rica. To achieve this, sensors (triaxial geophones) were placed in circular arrays and spectral ratios (from H/V) dispersion curves (from FK) and autocorrelation curves (from SPAC) for each site were obtained. Based on the above, the soil type was determined according to the characteristic site period $\left(\mathrm{T}_{0}\right.$ ) read from $\mathrm{H} / \mathrm{V}$ and criteria according to Zhao et al. (2006). Each site was also classified according to the Seismic Code of Costa Rica (2010) and Vs30 parameter calculated from the inversion of dispersion and autocorrelation curves. $T_{0}$ values from $0.3 \mathrm{~s}$ to $1.5 \mathrm{~s}$ and Vs30 between $244 \mathrm{~m} / \mathrm{s}$ to $379 \mathrm{~m} / \mathrm{s}$ were obtained, indicating that soils correspond to $\mathrm{S}_{3}$ (soft soil, $67 \%$ of all cases). No site whose classification corresponds to $\mathrm{S}_{1}$ (rock) or $\mathrm{S}_{4}$ (very soft soil) was found.

Keywords: Arrays, accelerometers, soils, surface waves, Vs30, Costa Rica.

RESUMEN: Se aplicaron las técnicas H/V, FK y SPAC, todas basadas en la medición de ondas superficiales generadas principalmente por vibraciones ambientales, para la caracterización de los suelos de 15 sitios seleccionados, 12 de ellos correspondientes a estaciones acelerográficas del Laboratorio de Ingeniería Sísmica de la Universidad de Costa Rica. Para este fin, se colocaron 8 sensores (geófonos triaxiales) en configuraciones circulares y se obtuvieron razones espectrales (H/V), curvas de dispersión (FK) y curvas de autocorrelación (SPAC) para cada sitio considerado. Con base en lo anterior, se determinó el tipo de suelo según el periodo característico del sitio ( $\mathrm{T}_{0}$ ) leído de H/V y según criterios de Zhao et al. (2006). También se clasificó cada sitio según el Código Sísmico de Costa Rica (2010) y el parámetro
\end{abstract}


Vs30 calculado a partir de la inversión de las curvas de dispersión y autocorrelación. Los valores de $\mathrm{T}_{0}$ varían entre $0,3 \mathrm{~s}$ a $1,5 \mathrm{~s}$ y las Vs 30 entre $244 \mathrm{~m} / \mathrm{s}$ a $379 \mathrm{~m} / \mathrm{s}$, lo que indica que los suelos que predominan en los sitios en estudio corresponden a $\mathrm{S}_{3}$ (suelo blando, un $67 \%$ de todos casos). No se encontró algún sitio cuya clasificación corresponda a $\mathrm{S}_{1}$ (roca) ni a $\mathrm{S}_{4}$ (suelo muy blando).

Palabras clave: Arreglos, acelerógrafos, suelos, ondas superficiales, Vs30, Costa Rica.

\section{INTRODUCCIÓN}

El Laboratorio de Ingeniería Sísmica (LIS) del Instituto de Investigaciones en Ingeniería de la Universidad de Costa Rica ha adquirido desde el 2010, más de 100 acelerógrafos nuevos que se encuentran conectados vía internet, lo que permite que la información registrada sea obtenida en tiempo real, por lo que es posible generar una gran cantidad de datos útiles para profesionales de distintas áreas (ingenieros, geólogos, geotecnistas, planificadores urbanos y encargados en atención de emergencias), pocos minutos después de haber ocurrido el evento.

Diversos investigadores alrededor del mundo coinciden en que es esencial conocer con exactitud el tipo de suelo existente en los sitios donde se registran los sismos, ya que de este dependerán parámetros muy importantes que se requieren para el diseño sismorresistente de estructuras, como lo son la aceleración pico, las pseudo aceleraciones, pseudo velocidades y desplazamientos espectrales, así como los espectros de diseño.

En vista de que en el pasado, el número promedio de estaciones con las que se contaba en el LIS eran alrededor de 30 y que muchos de los sitios actuales son nuevos, se desconoce con precisión el tipo de suelo que predomina en los emplazamientos donde tales instrumentos han sido colocados.

Existen numerosas técnicas y procedimientos que permiten realizar la caracterización de suelos, desde métodos sencillos y de bajo costo pero poco precisos, hasta otros muy sofisticados, pero complejos y costosos. Lo que sí resulta importante es escoger una técnica sistemática y uniforme que pueda ser aplicada en todos los sitios, de manera que se obtengan criterios comparables entre los distintos lugares en estudio, así como con condiciones de suelos estudiadas en otras partes del mundo.

En esta investigación se propone la aplicación de tres técnicas basadas en la medición de ondas superficiales llamadas H/V, FK y SPAC, que se describen brevemente en la metodología. Estas técnicas no son invasivas, ni destructivas del medio donde se aplican, son de fácil y rápida aplicación y tienen un costo relativamente bajo; por lo tanto, son muy convenientes para aplicar en la caracterización de sitios correspondientes a redes acelerográficas.

Las vibraciones ambientales, que están formadas predominantemente por ondas superficiales, son producidas por distintos tipos de fuente (SESAME European Research Project, 2004). Se dividen en dos grupos:

a- Antrópicas (generadas por humanos) como lo son la maquinaria y el tránsito vehicular, en un rango de frecuencias de $1 \mathrm{~Hz}$ a $10 \mathrm{~Hz}$ (alta frecuencia, ya que provienen de fuentes cercanas). También se les conoce con el nombre de microtremores o microtemblores.

b- Naturales, de origen oceánico y por condiciones ambientales, como el oleaje, el viento, etc. Tienen un rango de frecuencias predominante entre $0,1 \mathrm{~Hz}$ a $1 \mathrm{~Hz}$ (baja frecuencia ya que provienen de fuentes lejanas). Se conocen con el nombre de microsismos.

La principal ventaja de trabajar con este tipo de vibraciones es que están presentes casi todo el tiempo y en cualquier sitio, por lo que su medición puede llevarse a cabo en cualquier momento, con procedimientos relativamente sencillos. 


\section{METODOLOGÍA}

Para lograr una clasificación homogénea de los sitios, a cada estación seleccionada se le aplicaron los siguientes métodos:

-Técnica H/V: Consiste en la determinación de los espectros de Fourier de las componentes horizontales y de la vertical de registros de vibraciones ambientales, obtenidos con un velocímetro o un acelerógrafo triaxial para una ventana de tiempo total de aproximadamente media hora por cada sitio. A partir de estos espectros se obtiene la razón $\mathrm{H} / \mathrm{V}$ (cociente espectral), que es considerado por su autor (Nakamura, 1989) como la función de transferencia aproximada de los estratos del suelo sobre el basamento. Al contarse con dos componentes horizontales, $\mathrm{H}$ suele ser considerado como el promedio de los espectros en el plano horizontal.

El método $\mathrm{H} / \mathrm{V}$ parte de la hipótesis que las vibraciones ambientales están compuestas por varios tipos de ondas y considera que estas son similares horizontal y verticalmente en el basamento. Al propagarse por las distintas capas del suelo, las vibraciones en componente horizontal son amplificadas debido a multirreflexiones de la onda $\mathrm{S}$. Esto es válido si en estas vibraciones predominan ondas Rayleigh y su elipticidad es casi unitaria, lo que generalmente sucede en medios estratificados, donde hay un contraste significativo entre las impedancias de las capas de suelo y del basamento. El método ha sido ampliamente utilizado y se dispone de una exhaustiva lista de recomendaciones para su aplicación (SESAME European Research Project, 2004).
El pico máximo observado en la razón espectral corresponde al período fundamental de vibración del sitio $\left(\mathrm{T}_{0}\right)$.

Una vez identificado el periodo fundamental, es posible clasificar el sitio a partir de correlaciones ya establecidas, según el cuadro 1 .

Este método sirve como un procedimiento preliminar de clasificación y permite tener un criterio inicial sobre el tipo de suelo existente en cada emplazamiento.

En caso de que la razón espectral tenga una forma muy plana y con valores de $\mathrm{H} / \mathrm{V}$ cercanos a la unidad, se considerará que el sitio corresponde a condición de roca.

En el caso de la presente investigación, se aplicó la técnica utilizando los 8 instrumentos colocados al mismo tiempo para formar los arreglos o arrays. Esto permitió además verificar la horizontalidad de los distintos estratos de suelo (aproximadamente las mismas razones espectrales obtenidas en todos los sitios donde se colocó el arreglo), además de su heterogeneidad debido a la identificación de algún pico, lo que implica la presencia de al menos un contraste significativo de impedancias entre suelo y basamento (SESAME European Research Project, 2004).

Se trabajó con ventanas de tiempo de $30 \mathrm{mi}-$ nutos, que son divididas por el algoritmo de cálculo en longitudes de acuerdo con distintos criterios. En este caso en particular se trabajó con longitudes dependientes de la frecuencia $\mathrm{f}_{0}$, de manera que el número de $\mathrm{f}_{0}$ dentro de cada ventana fuera superior a 30 .

\section{Cuadro 1}

Correspondencia entre clases de sitio, $\mathrm{T}_{0}$ y rangos de velocidades Vs (Zhao et al. 2006)

\begin{tabular}{cccc}
\hline Clases de sitio & $\begin{array}{c}\text { Periodo natural } \\
\text { del sitio } \mathrm{T}_{0}(\mathrm{~s})\end{array}$ & $\begin{array}{c}\text { Promedio de las velocidades } \\
\text { de onda cortante }(\mathrm{m} / \mathrm{s})\end{array}$ & $\begin{array}{c}\text { Equivalencia con la } \\
\text { clasificación del NEHRP }\end{array}$ \\
\hline S I (roca/suelo rígido) & $\mathrm{T}_{0}<0,2$ & $\mathrm{Vs} 30>600$ & $\mathrm{~A}+\mathrm{B}$ \\
S II (suelo firme) & $0,2=\mathrm{T}_{0}<0,4$ & $300<\mathrm{Vs} 30=600$ & $\mathrm{C}$ \\
S III (suelo medio) & $0,4=\mathrm{T}_{0}<0,6$ & $200<\mathrm{Vs} 30=300$ & $\mathrm{D}$ \\
S IV (suelo blando) & $\mathrm{T}_{0}=0,6$ & $\mathrm{Vs} 30=200$ & $\mathrm{E}$ \\
\hline
\end{tabular}


La frecuencia fundamental o característica del sitio $\left(\mathrm{f}_{0}\right)$ o bien el periodo fundamental $\left(\mathrm{T}_{0}\right.$ $=1 / f_{0}$ ) se leyó como el correspondiente al pico máximo del promedio de las razones $\mathrm{H} / \mathrm{V}$.

Técnicas basadas en arrays (arreglos de sensores colocados en serie) para la aplicación de las metodologias FK (Frequency-Wavenumber) y SPAC (Spatial Autocorrelation)

El registro simultáneo de varios sensores distribuidos en una geometría determinada, conocido comúnmente como arrays, ha resultado ser un atractivo método para la determinación de las velocidades Vs y el espesor de los estratos del suelo a partir de mediciones de ruido hechas "in situ". Tiene la ventaja de no ser invasivo ni destructivo y de ser relativamente fácil de aplicar.

El método asume primeramente que las vibraciones ambientales están compuestas en forma mayoritaria por ondas superficiales y que la estructura del subsuelo está formada por estratos horizontales.

En medios unidimensionales y heterogéneos, las ondas superficiales son dispersivas y muestran variaciones de la velocidad aparente según la frecuencia. Los modos de ondas Love (SH) y Rayleigh (P-SV) coexisten en las componentes horizontales, mientras que las verticales son afectadas únicamente por ondas superficiales Rayleigh (Wathelet, 2007).

El método de número de onda vs. frecuencia, conocido como FK, asume que las ondas planas atraviesan el array que se ubica en la superficie del suelo. Considerando una onda de frecuencia $\mathrm{f}$ con una dirección de propagación y una velocidad conocidas (o los números de onda equivalentes $\mathrm{k}_{\mathrm{x}}$ y $\mathrm{k}_{\mathrm{y}}$ a lo largo de los ejes horizontales $\mathrm{X}$ y Y), los tiempos de arribo son calculados en todos los sensores según su ubicación y las fases de los registros son cambiadas según los tiempos de retardo. La respuesta del array es calculada sumando las señales transformadas al dominio de la frecuencia. Si las ondas viajan con velocidad y dirección específicas, todas las contribuciones se acumularán constructivamente, resultando un array de gran salida o respuesta. La localización de esta máxima salida en el plano $\mathrm{k}_{\mathrm{x}} \mathrm{y} \mathrm{k}_{\mathrm{y}}$ (conocida también como beam power), provee una estimación de la velocidad y del azimut de las ondas que viajan a través del array (Wathelet, 2007).

Por otro lado, el método de la autocorrelación espacial (SPAC) representa otra posibilidad para analizar las señales registradas, el cual asume la distribución de las fuentes en el campo de ondas del ruido como aleatoria, tanto en el tiempo como en el espacio. En el caso de una onda dispersiva, se ha demostrado que las razones de autocorrelación son función de la fase de las velocidades y de la apertura del array. Este método aprovecha entonces la distribución aleatoria de las fuentes para relacionar las razones de autocorrelación con las velocidades de fase (Wathelet, 2007) .

Las siguientes imágenes muestran los sensores y el digitalizador utilizado para realizar las mediciones (Fig 1).

Para la aplicación de las técnicas FK y SPAC, se colocaron en la mayoría de los casos 8 sensores en una superficie horizontal, normalmente en canchas de futbol que son los espacios abiertos y horizontales que predominan en el país (Fig. 2).

Un sensor es ubicado en un punto central y siete perimetrales, ya que debe lograrse una adecuada cobertura acimutal. Debido a que también deben registrarse adecuadamente los frentes de onda en términos radiales, se hicieron mediciones considerando dos radios por sitio, de manera que el menor defina las capas superficiales (altas frecuencias de la curva de dispersión) y que el radio mayor defina las capas más profundas (frecuencias bajas o periodos largos de la misma curva). 


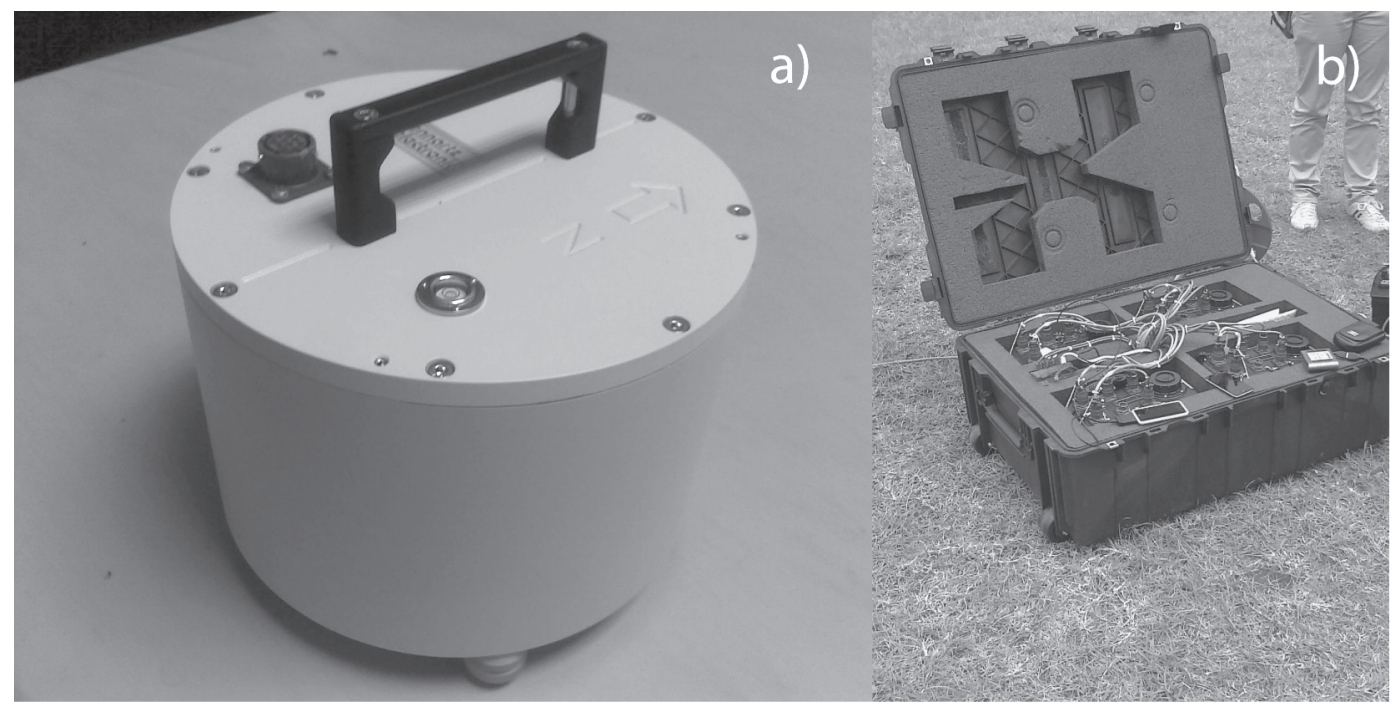

Fig. 1: a) Fotografía de los sensores Lennartz de 20 segundos (8 en total) y b) del digitalizador integrado Reftek (modelo SMA) utilizado.

Las longitudes máximas disponibles de los cables son de $50 \mathrm{~m}$, lo que condiciona la apertura máxima del arreglo debido a que todos los sensores están conectados a un único digitalizador ubicado en el centro del arreglo. Sin embargo, las limitaciones usuales de los espacios abiertos existentes para aplicar estas técnicas coinciden con estas aperturas. Los sensores se colocaron a partir de mediciones hechas con cintas métricas.

Sobre los sensores se colocaron unas cajas plásticas y sobre ellas un peso superior, de manera que se eviten vibraciones del sensor debidas al viento.

La figura 2 muestra la disposición de los sensores en la cancha de futbol de Palmares, así como un esquema en planta de la ubicación de los mismos.

Una vez realizadas las mediciones de $30 \mathrm{mi}$ nutos en cada sitio, se procesaron los registros de ruido semejantes a los obtenidos en la figura 3 . En la fig. 3a se observan registros típicos, donde es claro notar los arribos de los distintos frentes de ondas, señalados en líneas verticales. En el caso mostrado en la fig. 3b, es menos sencillo identificar frentes de onda comunes a todos los registros, ya que aparecen picos específicos debidos a vibraciones muy locales, que no son registrados por todos los sensores. Los picos que no son registrados simultáneamente por todos los geófonos son descartados por el programa de cálculo.

Con los registros de ruido, se procedió a generar las curvas de dispersión (método FK) o las curvas de autocorrelación (método SPAC) para cada sitio con el uso del programa de libre distribución GEOPSY. Un ejemplo de estas se muestra en la figura 4, para el sitio Fabio Baudrit, ubicado en Alajuela. En la figura 4a (método FK) el eje Y corresponde a la lentitud (o inverso de la velocidad aparente) y en el X se observa la frecuencia asociada a cada velocidad, lo que es típico en medios heterogéneos y por lo tanto dispersivos. En la figura $4 \mathrm{~b}$ (método SPAC) se presenta la curva de dispersión obtenida a partir de las curvas de autocorrelación. 

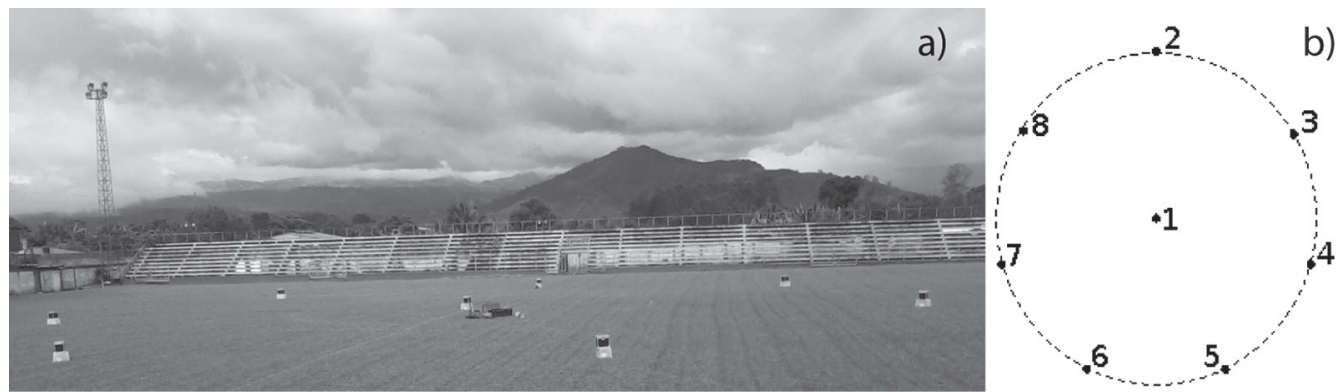

Fig. 2: a) Ubicación de sensores en una cancha de futbol en el estadio de Palmares. b) Disposición circular en planta de 8 sensores, cuyo radio suele variar entre 30 y $50 \mathrm{~m}$ (radio grande) y entre 15 y $25 \mathrm{~m}$ (radio pequeño).

La última etapa para obtener los modelos de Vs vs. profundidad, consiste en invertir la curva de dispersión.

Este es un procedimiento "a la inversa" en el sentido de que lo usual es que a partir de un medio estratificado y de un registro de entrada en la base del mismo, se estime la respuesta en superficie. En esta investigación, se cuenta con los registros en superficie que atravesaron el perfil de suelos (registros de ruido), por lo que ellos contienen

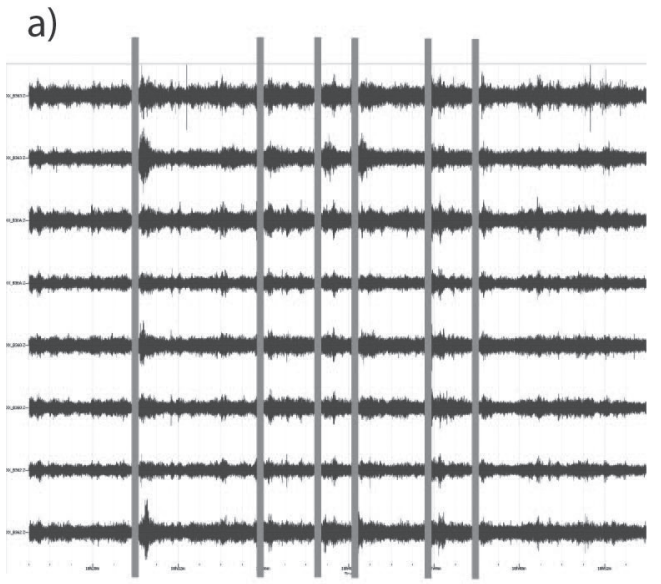

toda la información de los estratos por donde viajaron los frentes de onda hasta llegar a la superficie. De ellos se estiman las curvas de dispersión y estas se invierten, generándose en forma iterativa varios modelos que representan al medio, y cuya selección final corresponde al que muestre menor error (una mejor aproximación entre la curva de dispersión dada y la generada por el modelo). Para esta investigación no se utilizó información adicional obtenida por otras fuentes, debido a que no fue fácil su localización. b)

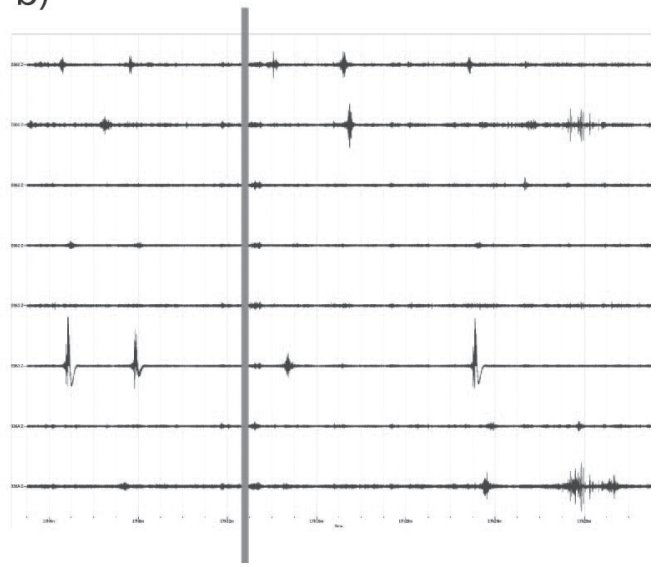

Fig. 3: Gráfico de señales de ruido típicas. En a) se observan varios frentes de onda registrados por todos los sensores (líneas verticales) y en b) solo se aprecia un frente claro. 
a)

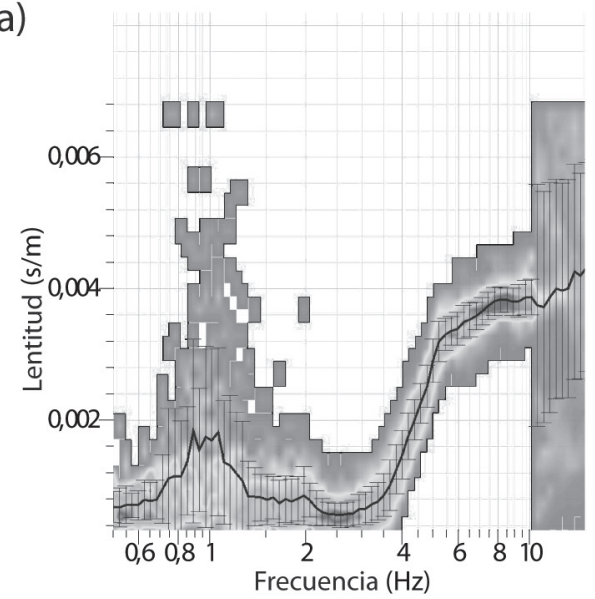

b)

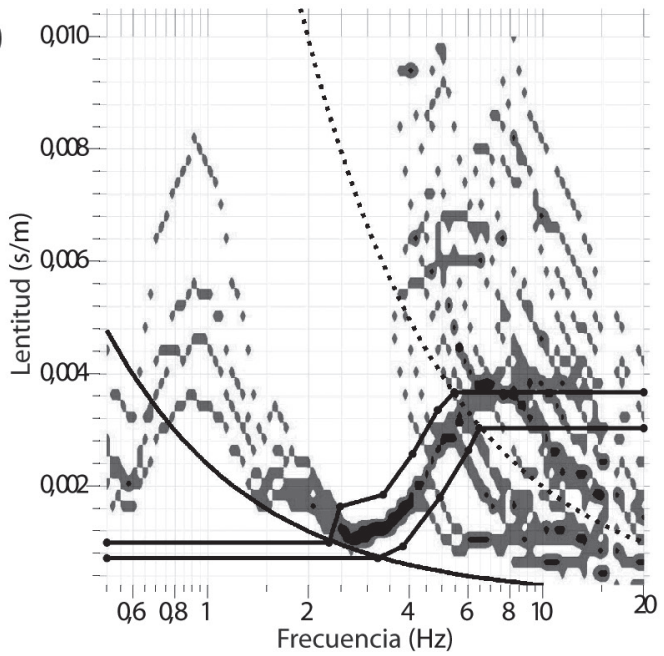

Fig. 4: a) Curva de dispersión obtenida con el método FK a partir de las mediciones de vibraciones realizadas en la estación Fabio Baudrit, Alajuela. b) Curva de dispersión obtenida a partir del método de autcorrelación (SPAC) para el mismo sitio.

Las figuras $5 \mathrm{a}$ y $5 \mathrm{~b}$ muestran los resultados obtenidos para los sitios de plaza de Derecho UCR y plaza Iglesias de Cartago, respectivamente. Las líneas punteadas corresponden a los modelos más apropiados para cada sitio, ya que son los que muestran menor error (mejor aproximación a la curva de dispersión dada).

Una vez obtenido el perfil de velocidades, se estimó el parámetro Vs30, corresponde al promedio ponderado de velocidades de ondas de corte correspondiente a los primeros $30 \mathrm{~m}$ medidos desde la superficie. Su expresión matemática es:

$$
V s 30=\frac{\sum_{i=1}^{N} d_{i}}{\sum_{i=1}^{N} \frac{d_{i}}{V_{s i}}}
$$

Siendo "di" el espesor de cada capa de suelo del perfil hasta alcanzar los $30 \mathrm{~m}$ de profundidad, "Vsi" la velocidad de onda cortante de cada capa i en $\mathrm{m} / \mathrm{s}$ y $\mathrm{N}$ el número de capas hasta alcanzar los $30 \mathrm{~m}$. Una gran ventaja de Vs30 es que muchos de los suelos en el mundo han sido clasificados a partir de este parámetro, por lo que se facilita en gran medida su uso y comparación con estudios que provengan de distintos lugares. Por otro lado, la Vs30 permite tener información del tipo de suelo predominante en un sitio hasta los $30 \mathrm{~m}$ de profundidad, evitándose suposiciones inadecuadas al caracterizar el suelo si se considera solamente la geología superficial observada en el sitio o en mapas, como solía hacerse en el pasado.

Es posible obtener la clasificación de suelos para cada estación según la correspondencia mostrada en el cuadro 2, que son las propuestas por los códigos NEHRP, mundialmente utilizado como referencia (BSSC, 2003) y Código Sísmico de Costa Rica CSCR-2010 (CFIA, 2010). Se observa que para el caso del CSCR-2010 no existe la clasificación de "roca dura" ya que es muy poco probable encontrarla en superficie en el territorio nacional. 
a)

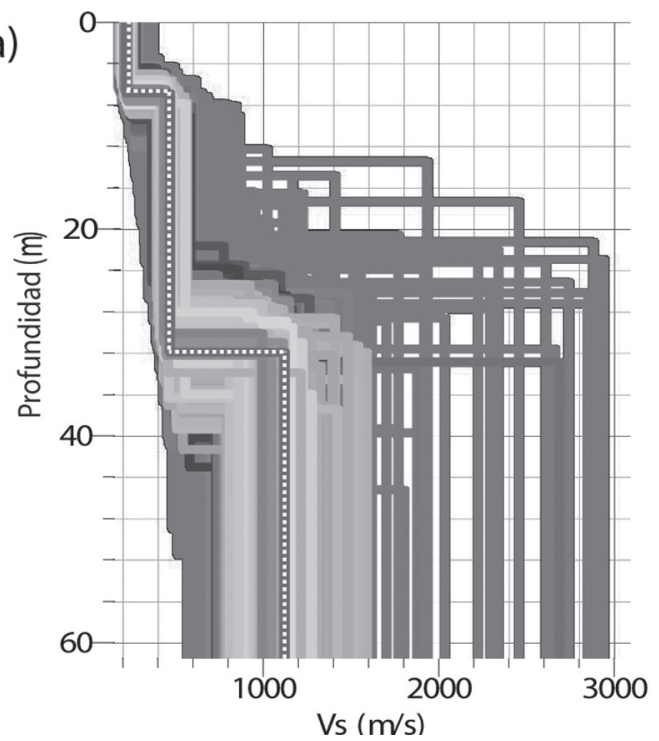

b)

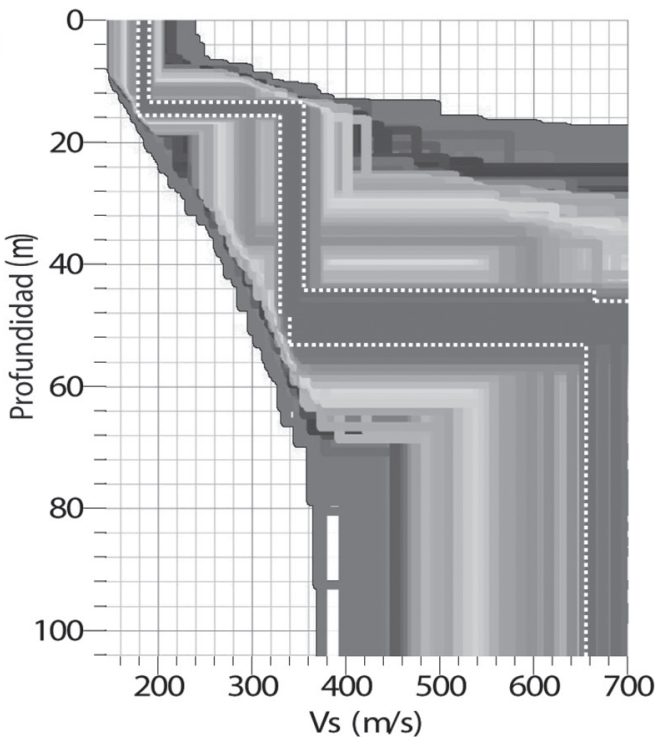

Fig. 5: a) Modelos de velocidad de onda cortante vs. profundidad obtenidos a partir de métodos de inversión, con mediciones hechas en la plaza al lado de Derecho, UCR y b) en la plaza Iglesias de Cartago. Las líneas punteadas corresponden a los mejores modelos obtenidos.

Si solamente se conoce el periodo fundamental del sitio, Zhao et al. (2006) proponen un método de clasificación basado en el cuadro 1 y utilizado ampliamente en Japón.

Una vez obtenido $\mathrm{T}_{0}$ con el método $\mathrm{H} / \mathrm{V}$ y la clasificación de suelo asociada, así como el parámetro Vs30 y el tipo de suelo correspondiente, se creó una tabla en la que se incluyen todos los criterios empleados en este estudio a partir de los cuales se definió el tipo de suelo de cada emplazamiento. De este modo se logra obtener una clasificación homogénea y consistente.

\section{RESULTADOS}

En el cuadro 3 se enlistan los sitios donde se realizaron las pruebas, la fechas de las mismas, el número de sensores utilizados y los diámetros según cada sitio, siguiendo siempre configuraciones circulares recomendadas por Aki, (1957) ya que permiten una mejor cobertura radial y acimutal del registro de los frentes de onda. En total se analizaron 15 sitios, que corresponden a 12 estaciones acelerográficas (los 12 primeros sitios de la tabla con el nombre de la estación entre paréntesis) y los tres últimos fueron sitios de pruebas.

La figura 6 muestra las tres distintas configuraciones usadas, según el número de sensores disponible. La configuración ideal es la de 8 (uno central y 7 perimetrales). Sin embargo, el octavo sensor se adquirió aproximadamente un año después de los demás, por lo que las primeras mediciones se hicieron con 7 y 6 sensores, siempre con uno central. La configuración de 7 sensores (fig. 6b) no es la más recomendada ya que implica que tres sensores se alinean a la vez, lo que no permite una buena cobertura acimutal de los frentes de 


\section{Cuadro 2}

Clasificación de suelos con base en los códigos NEHRP (BSSC, 2003) y CSCR-2010 (CFIA, 2010)

\begin{tabular}{|c|c|c|c|c|c|}
\hline \multicolumn{3}{|c|}{ NEHRP } & \multicolumn{3}{|c|}{ CSCR-2010 } \\
\hline Clase de suelo & $\begin{array}{l}\text { Características } \\
\text { del suelo }\end{array}$ & $\begin{array}{l}\text { Rango Vs30 } \\
(\mathrm{m} / \mathrm{s})\end{array}$ & Clase de suelo & $\begin{array}{l}\text { Características d } \\
\text { el suelo }\end{array}$ & $\begin{array}{c}\text { Rango Vs30 } \\
(\mathrm{m} / \mathrm{s})\end{array}$ \\
\hline A & Roca dura & $\mathrm{Vs} 30>1500$ & & & \\
\hline B & Roca & $760<\mathrm{Vs} 30 \leq 1500$ & S1 & $\begin{array}{c}\text { Roca o suelo rígido o } \\
\text { denso }\end{array}$ & $\geq 760$ \\
\hline $\mathrm{C}$ & $\begin{array}{l}\text { Suelo muy denso y roca } \\
\text { suave }\end{array}$ & $360<\mathrm{Vs} 30 \leq 760$ & S2 & $\begin{array}{l}\text { Medianamente a denso } \\
\text { a denso o mediana- } \\
\text { mente rígido a rígido }\end{array}$ & 350 a 760 \\
\hline $\mathrm{D}$ & Suelo firme a medio & $180 \leq \mathrm{Vs} 30 \leq 360$ & $\mathrm{~S} 3$ & $\begin{array}{l}6 \text { a } 12 \text { m de arcilla de } \\
\text { consistencia de suave a } \\
\text { medianamente rígida }\end{array}$ & 180 a 350 \\
\hline $\mathrm{E}$ & Suelo blando & Vs $30<180$ & S4 & $\begin{array}{l}\text { Más de } 12 \mathrm{~m} \text { de arcilla } \\
\text { suave }\end{array}$ & $\leq 180$ \\
\hline
\end{tabular}

Cuadro 3

Sitios donde se realizaron las mediciones y especificaciones de cada prueba

\begin{tabular}{|c|c|c|c|c|}
\hline Lugar & Día de la prueba & Número de sensores & Diám. ext (m) & Diám. int. (m) \\
\hline Alajuela, Plaza Acosta (AALA) & 1 agosto 2012 & 8 ( 1 central y 7 externos) & 70 & 35 \\
\hline Fabio Baudrit, UCR (AFBR) & 30 julio 2012 & 8 ( 1 central y 7 externos $)$ & 66 & 33 \\
\hline Grecia, Tacares UCR (AGRE) & 16 mayo 2012 & 6 ( 1 central y 5 externos $)$ & 74 & 37 \\
\hline Palmares, estadio (APMR) & 31 octubre 2012 & 8 ( 1 central y 7 externos $)$ & 84 & 42 \\
\hline San Ramón, UCR (ASRM) & 29 octubre 2012 & 8 ( 1 central y 7 externos) & 82 & 41 \\
\hline Cartago, Biblioteca (CCTR) & 31 julio 2012 & 8 ( 1 central y 7 externos $)$ & 50 & 25 \\
\hline Paraíso, cancha futbol (CPAR) & 8 febrero 2013 & 8 ( 1 central y 7 externos $)$ & 60 & 30 \\
\hline Cartago, Tecnológico (CTEC) & 31 enero 2013 & 8 ( 1 central y 7 externos) & 90 & 45 \\
\hline Hatillo, plaza futbol (SHTH) & 27 junio 2013 & 8 ( 1 central y 7 externos) & 66 & 33 \\
\hline Sabana, cancha beisbol (SSBN) & 12 junio 2013 & 8 ( 1 central y 7 externos) & 92 & 46 \\
\hline Santa Ana, St. Jude (SJUD) & 4 febrero 2013 & 8 ( 1 central y 7 externos) & 60 & --- \\
\hline Derecho, plaza UCR (SLPF) & 21 junio 2012 & 8 ( 1 central y 7 externos) & 50 & 25 \\
\hline Ing. Eléctrica, UCR & 1 febrero 2013 & 8 ( 1 central y 7 externos) & 50 & 25 \\
\hline Estadio ecológico, UCR & 27 febrero 2012 & 7 ( 1 central y 6 externos) & 66 & 33 \\
\hline Cartago, Plaza Iglesias & 26 julio 2012 & 6 ( 1 central y 5 externos $)$ & 73 & 36,5 \\
\hline
\end{tabular}




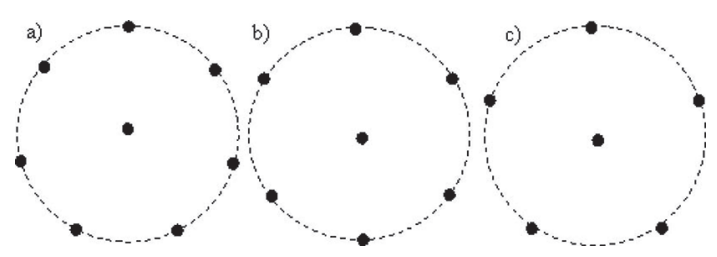

Fig. 6: Disposición de sensores según número: a) uno central y siete perimetrales (externos), b) uno central y seis perimetrales y c) uno central y cinco perimetrales.

onda. También se optó en algunos casos por trabajar con 6 sensores, que corresponden a uno central y cinco perimetrales, que al ser un número impar, no permite la alineación de más de dos sensores, lo cual es favorable.

El sitio donde se logró una mayor apertura (diámetro) es SSAB (Sabana), por lo que fue el que aportó mayor información de capas profundas. Los casos de SLPF (cancha Derecho) e Ingeniería Eléctrica de la UCR corresponden a los de menor apertura del arreglo; sin embargo, en ellos se trabajó con 8 sensores, lo que mejora la cobertura de los frentes de onda.

Las figuras 7 a 21 muestran los resultados obtenidos para todos los sitios. Se presenta en la esquina superior izquierda la razón espectral $\mathrm{H} / \mathrm{V}$, en la superior derecha las curvas de dispersión obtenidas con las técnicas FK y SPAC y en la parte inferior de las figuras los modelos de Vs vs. profundidad obtenidos por esos dos métodos (inferior izquierda corresponde a FK e inferior derecha a SPAC). A continuación se hacen breves comentarios por sitio:

Alajuela Plaza Acosta (Fig. 7). La razón espectral $\mathrm{H} / \mathrm{V}$ define en forma clara un único pico en $1,42 \mathrm{~Hz}$, por lo que se considera la presencia de un solo contraste importante entre capas. Las tendencias de las curvas de dispersión obtenidas con los métodos FK y SPAC son semejantes, aún cuando SPAC aporta mayor información de bajas frecuencias y FK en altas. Tanto el mejor modelo con FK como el de SPAC dan resultados semejantes: dos capas y el basamento, presentándose el mayor contraste entre la segunda capa y el basamento., a unos 40 a $45 \mathrm{~m}$ de profundidad.

Alajuela Fabio Baudrit, UCR (Fig. 8). Con la razón $\mathrm{H} / \mathrm{V}$ se define bien un único pico en 2,07 $\mathrm{Hz}$. Las tendencias de las curvas de dispersión obtenidas con FK y SPAC y los modelos obtenidos por los dos métodos son semejantes: una sola capa con un único contraste entre 28 a $30 \mathrm{~m}$, aunque en frecuencias bajas el método FK no resuelve de manera efectiva como sí lo hace SPAC.

Tacares de Grecia, UCR (Fig. 9). Se observa dispersión entre las razones $\mathrm{H} / \mathrm{V}$ (dificultad para la identificación de la frecuencia fundamental $\mathrm{f}_{0}$ ). Además, se notan valores de amplificación muy altos en bajas frecuencias, lo que puede estar asociado a la presencia de viento y a un aislamiento incorrecto del sensor a este fenómeno. La curva de dispersión obtenida con FK es casi horizontal a partir de $7 \mathrm{~Hz}$, situación que no se observa en la obtenida con SPAC. Ambas son concordantes entre 3 y $7 \mathrm{~Hz}$. Respecto a los modelos, hay bastante dispersión en ambos casos, sobre todo para SPAC, además de que se generaron pocos modelos aceptables (mostrados cerca de la línea de puntos). En FK se presenta un contraste a los $22 \mathrm{~m}$ y otro más significativo a los $60 \mathrm{~m}$, aproximadamente.

Estadio de Palmares, Alajuela (Fig. 10). Se definen varios picos en todas las razones $\mathrm{H} / \mathrm{V}$, sobre todo en 5 y en $0,5 \mathrm{~Hz}$ que es el de mayor amplitud, lo que puede representar el máximo contraste entre capas. En las curvas de dispersión se observa una disminución de las lentitudes en $5 \mathrm{~Hz}$, lo que puede estar asociado a una inversión de velocidades (Vs mayor en una capa superior). Sin embargo, esto no se observa en los modelos obtenidos con FK, donde si es clara la presencia de una capa con una Vs muy baja hasta los 10 de profundidad. En el caso de SPAC, si 

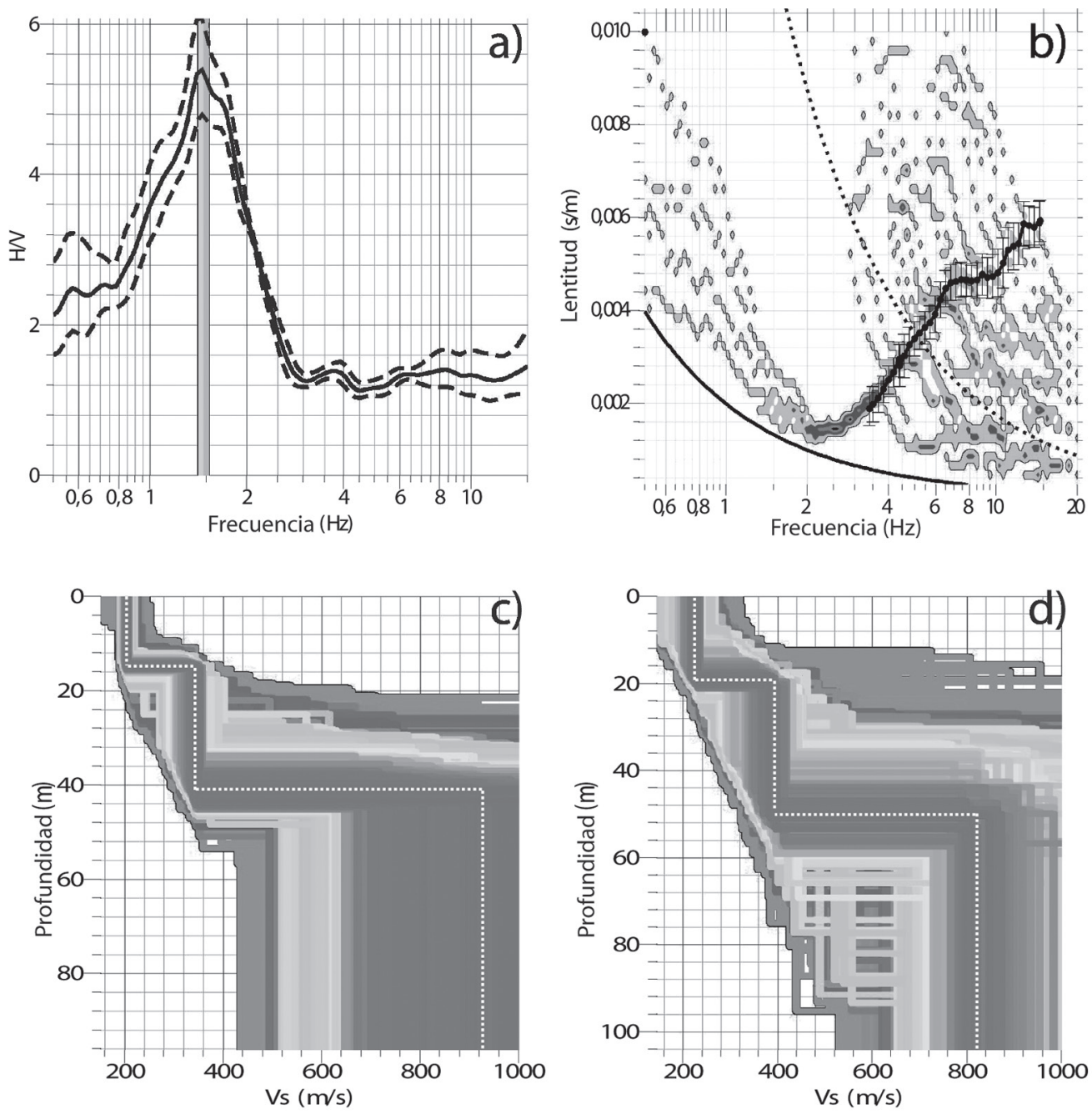

Fig. 7: Alajuela Plaza Acosta (cercana a AALA).

parece reconocerse una capa superficial con velocidad invertida en los primeros modelos, pero en las interaciones que muestran menor error esto desaparece. Es un sitio en el que se recomienda un análisis más detallado.

Pista de atletismo San Ramón, UCR (Fig. 11). Se observa en forma clara un pico en 0,95 $\mathrm{Hz}$, manifestado tanto en todos los sensores, tanto individualmente como en el promedio. La curva de dispersión obtenida con FK se vuelve casi 

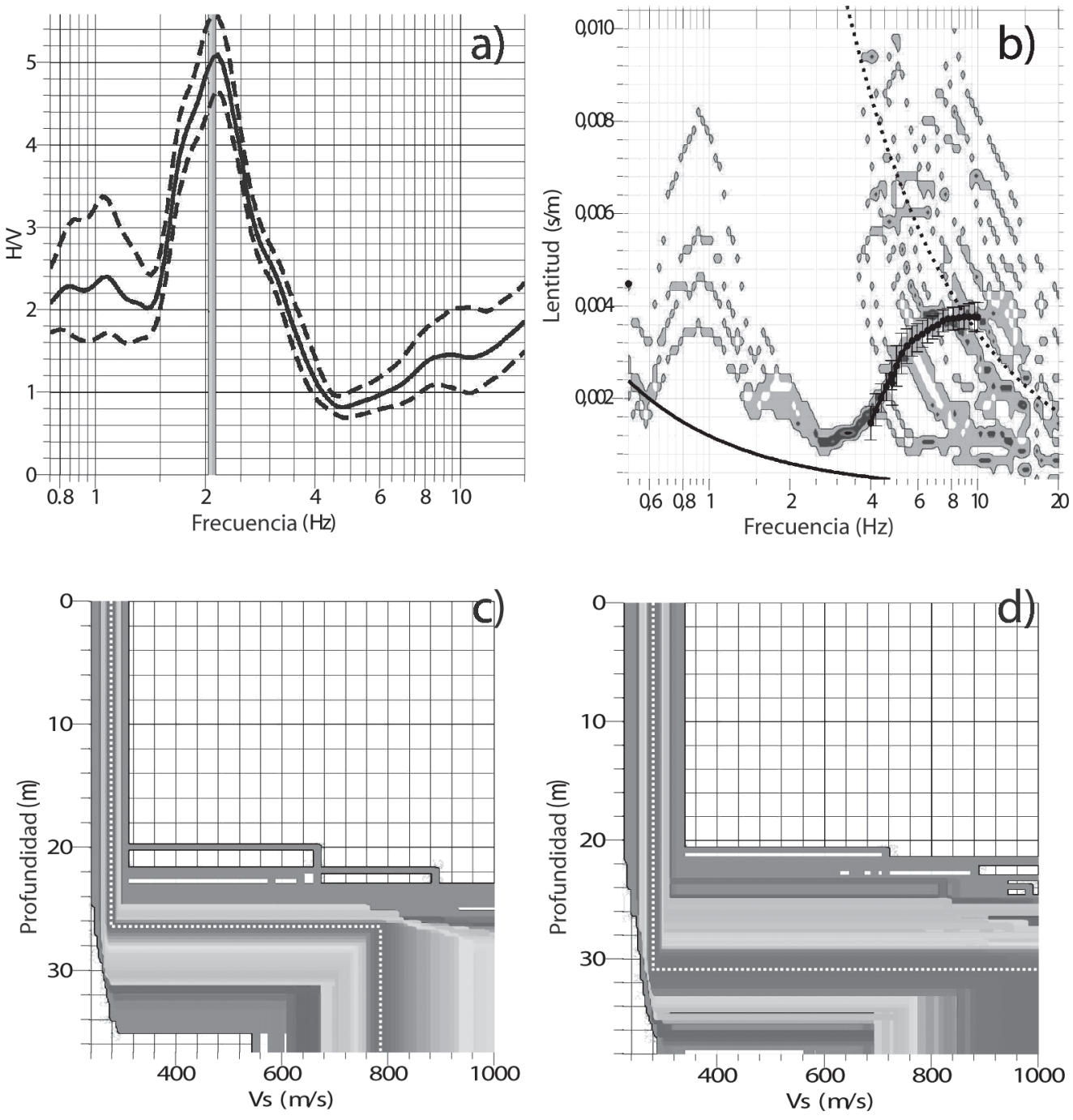

Fig. 8: Alajuela Fabio Baudrit, UCR (cercano a estación AFBR).

horizontal a partir de $3 \mathrm{~Hz}$ en un valor de lentitud cercano a $0,004 \mathrm{~s} / \mathrm{m}$ (velocidad de $250 \mathrm{~m} / \mathrm{s}$ ), que de acuerdo con los modelos de una capa obtenidos tanto con FK como SPAC, esta es aproximadamente la Vs menor encontrada. Luego, se manifiesta otro contraste significativo a partir de los $50 \mathrm{~m}$ en FK y otro a los $70 \mathrm{~m}$ según SPAC.

Plaza al lado de biblioteca, Cartago (Fig 12). Es clara cierta dispersión de los resultados de $\mathrm{H} / \mathrm{V}$ en la zona donde se ubica la frecuencia de máxima amplificación (aproximadamente en 0,9 $\mathrm{Hz}$ ). La curva de dispersión muestra una inusual forma "dentada", tanto con FK como con SPAC. Los modelos obtenidos con ambos métodos (FK 

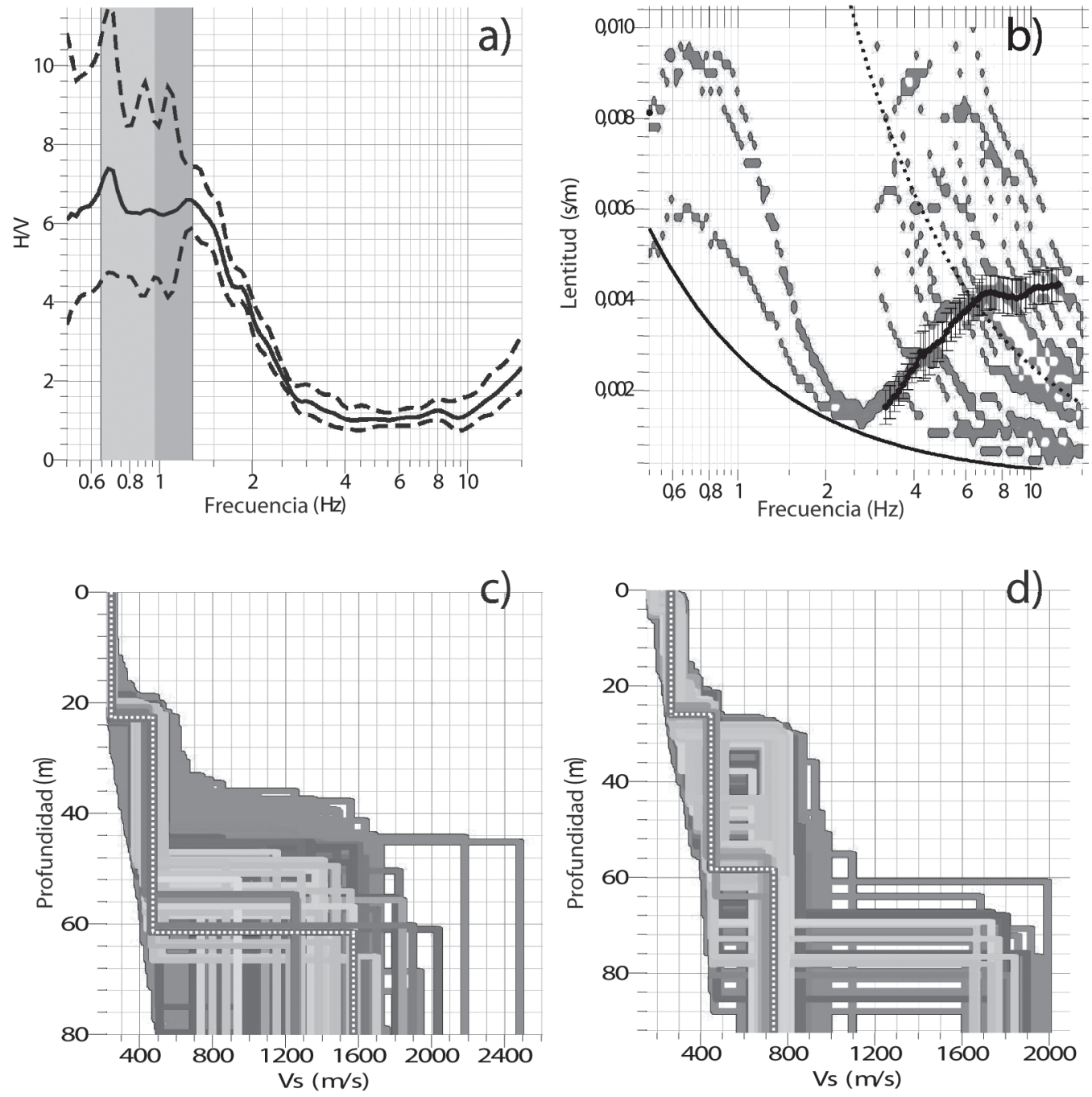

Fig. 9: Tacares de Grecia, UCR. (cerca de AGRE).

y SPAC) muestran resultados semejantes respecto a las profundidades de las capas pero un poco distintos respecto a los valores de Vs. La velocidad de onda cortante definida para el basamento también difiere. Esto puede deberse a que la no horizontalidad de los estratos observada en $\mathrm{H} / \mathrm{V}$, dificulta obtener resultados concluyentes acerca de los valores reales de Vs.
Plaza en Paraíso, Cartago (Fig. 13). Hay una clara dispersión y por lo tanto dificultad para leer la frecuencia fundamental en la razón $\mathrm{H} / \mathrm{V}$, principalmente en frecuencias bajas, pero en promedio se observa cercana a $0,9 \mathrm{~Hz}$. Las curvas de dispersión difieren para frecuencias inferiores a $4 \mathrm{~Hz}$ y se asemejan entre 4 y $8 \mathrm{~Hz}$. Se obtienen modelos 

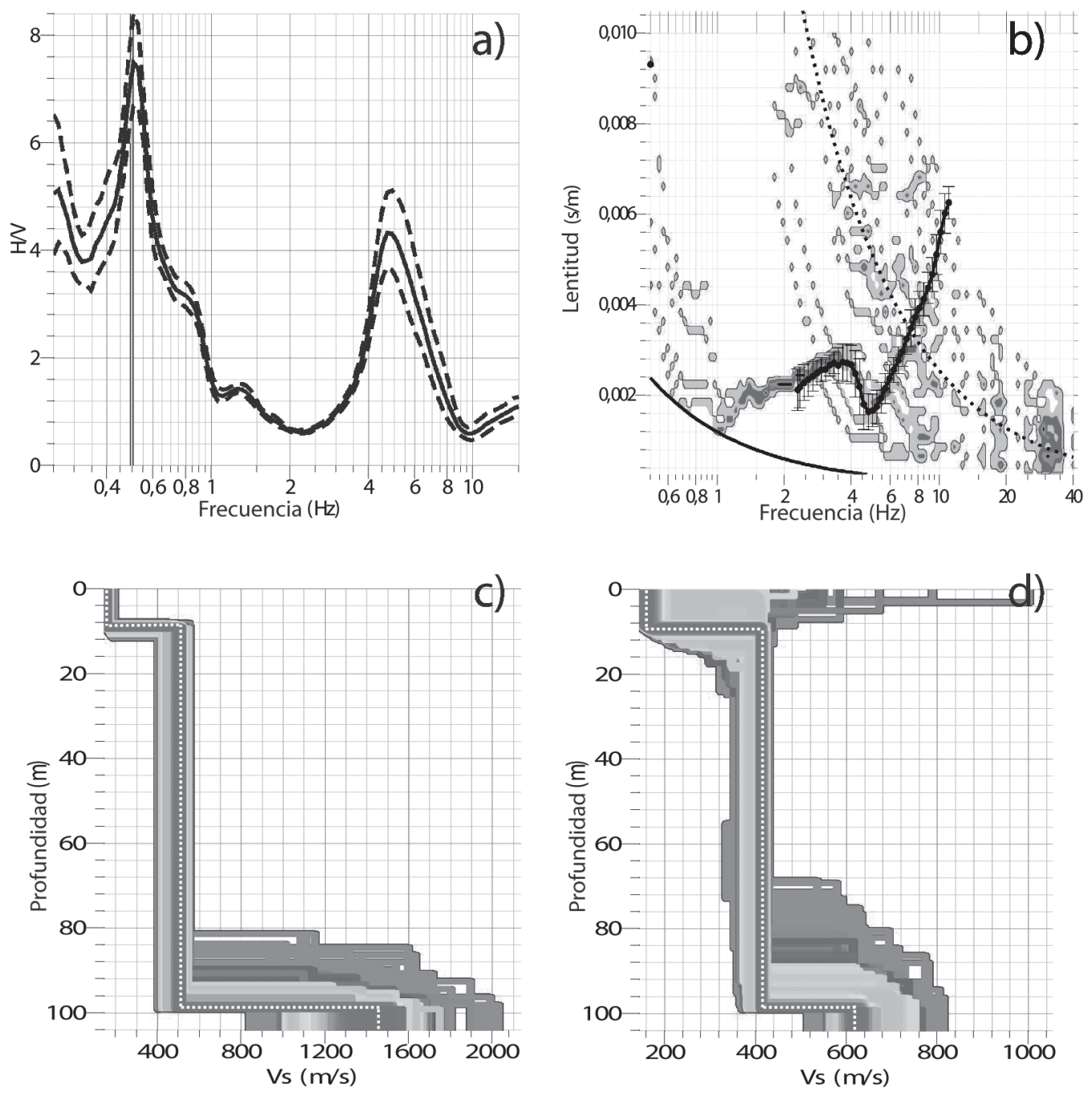

Fig. 10: Estadio de Palmares, Alajuela (cerca de estación APMR).

semejantes de 2 capas usando FK y SPAC, sobre todo en el primer contraste, tanto para la velocidad Vs1 como para la profundidad h1.

Cancha para beisbol, TEC, Cartago (Fig. 14). $\mathrm{H} / \mathrm{V}$ muestra bastante dispersión en amplitud y varios picos; sin embargo, se logra observar la frecuencia predominante del sitio en aproximadamente en $0,85 \mathrm{~Hz}$. El decaimiento de la curva de dispersión en frecuencias parece indicar la posible inversión de velocidades (una Vs mayor en una capa inferior). Esto parece mostrarse en los modelos obtenidos según el método FK, donde se 

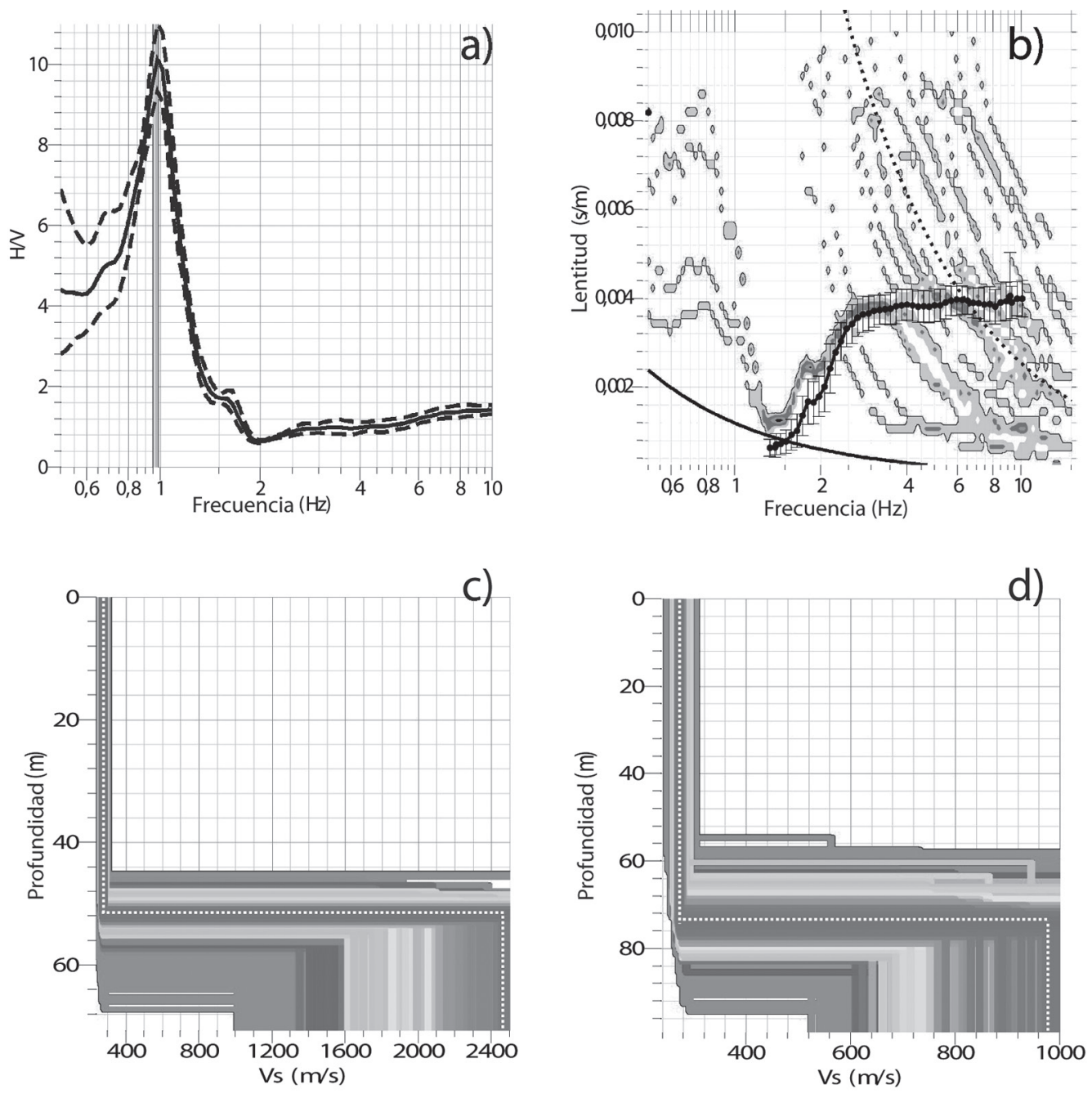

Fig. 11: Pista de atletismo San Ramón, UCR (cerca de estación ASRM).

definen varias capas. Sin embargo, el modelo estimado por SPAC, que es de una sola capa, muestra solamente un contraste significativo a los $45 \mathrm{~m}$ de profundidad. El método FK muestra una curva de dispersión bien definida y con desviaciones estándar bajas (fig. 14b), lo que parece indicar una mejor calidad de datos, además de que es el único que logra representar la inversión mencionada.
Plaza en Hatillo, San José (Fig. 15). En H/V se observan varios picos, cada uno probablemente asociado a un contraste distinto (cambio de suelo), siendo el más importante, correspondiente a la frecuencia fundamental, en aproximadamente 2,7 Hz. Las curvas de dispersión se asemejan en casi todo el rango de frecuencias, 

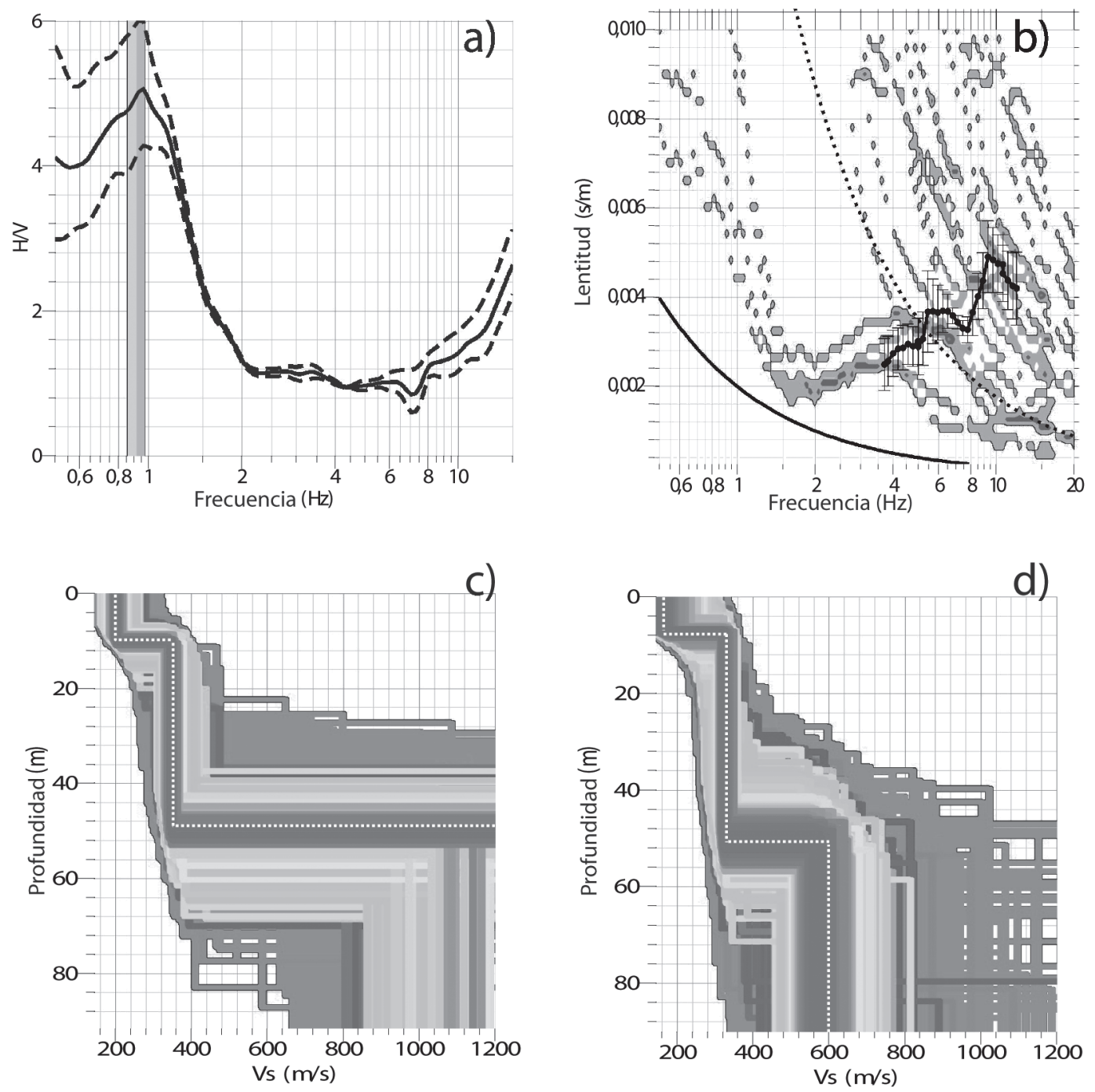

Fig. 12: Plaza al lado de biblioteca, Cartago (cerca de estación CCRT).

siendo bastante horizontales desde 1,5 hasta 7 $\mathrm{Hz}$ y luego hay un incremento (aumento de pendiente). Los modelos generados inician en forma semejante, definiendo 2 capas y un basamento. Sin embargo, los mejores resultados para cada método quedan representados por 2 capas para FK y 1 capa para SPAC. De acuerdo con el número de picos observados en $\mathrm{H} / \mathrm{V}$, tiene más sentido el modelo de un mayor número de capas, que representa varios contrastes. 

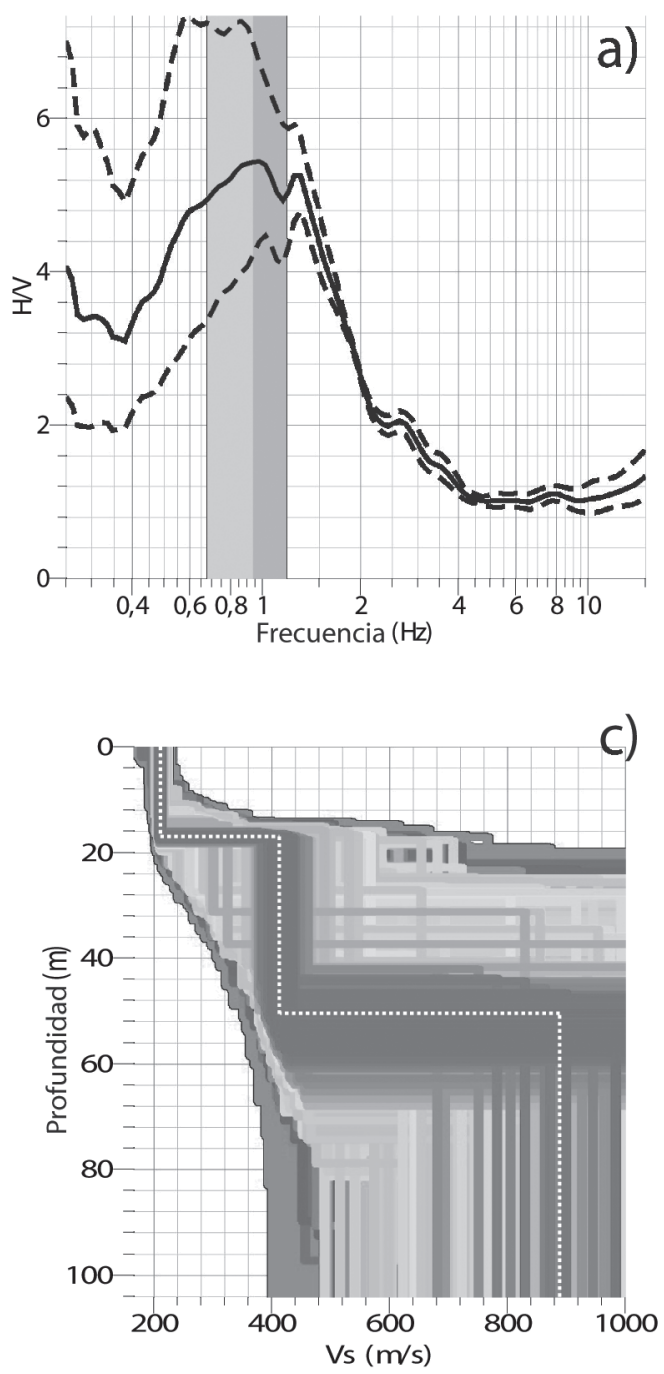

Fig. 13: Plaza en Paraíso, Cartago (cerca de estación CPAR).

Cancha de softball, La Sabana (Fig. 16). Es evidente el pico mostrado y bien definido por $\mathrm{H} / \mathrm{V}$ en 2 $\mathrm{Hz}$, lo que demuestra un claro contraste a los $40 \mathrm{~m}$ aprox. La curva de dispersión obtenida por SPAC no es clara, sin embargo, la generada por FK lo es en altas
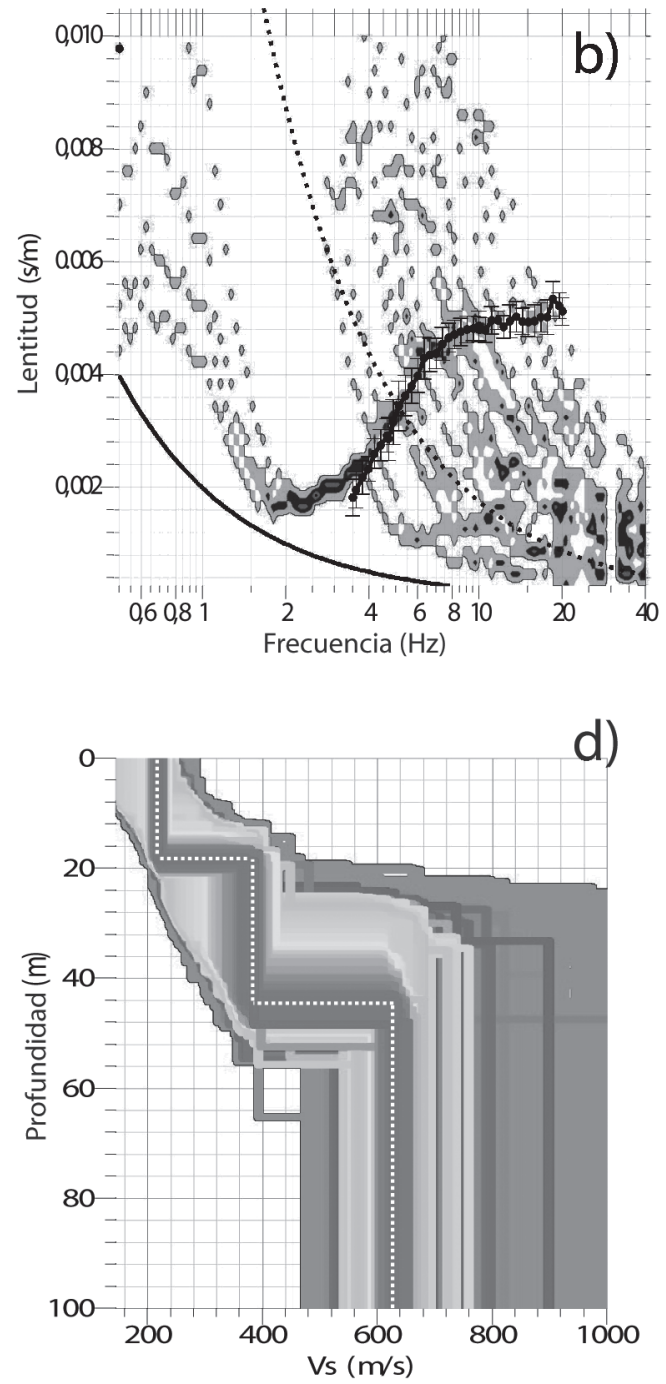

frecuencias, lo que genera un modelo de 2 capas y un basamento. La primera capa y el primer contraste están muy bien definidos hasta los $16 \mathrm{~m}$, luego una segunda Vs y contraste asociado que muestran alguna dispersión y finalmente el basamento. 

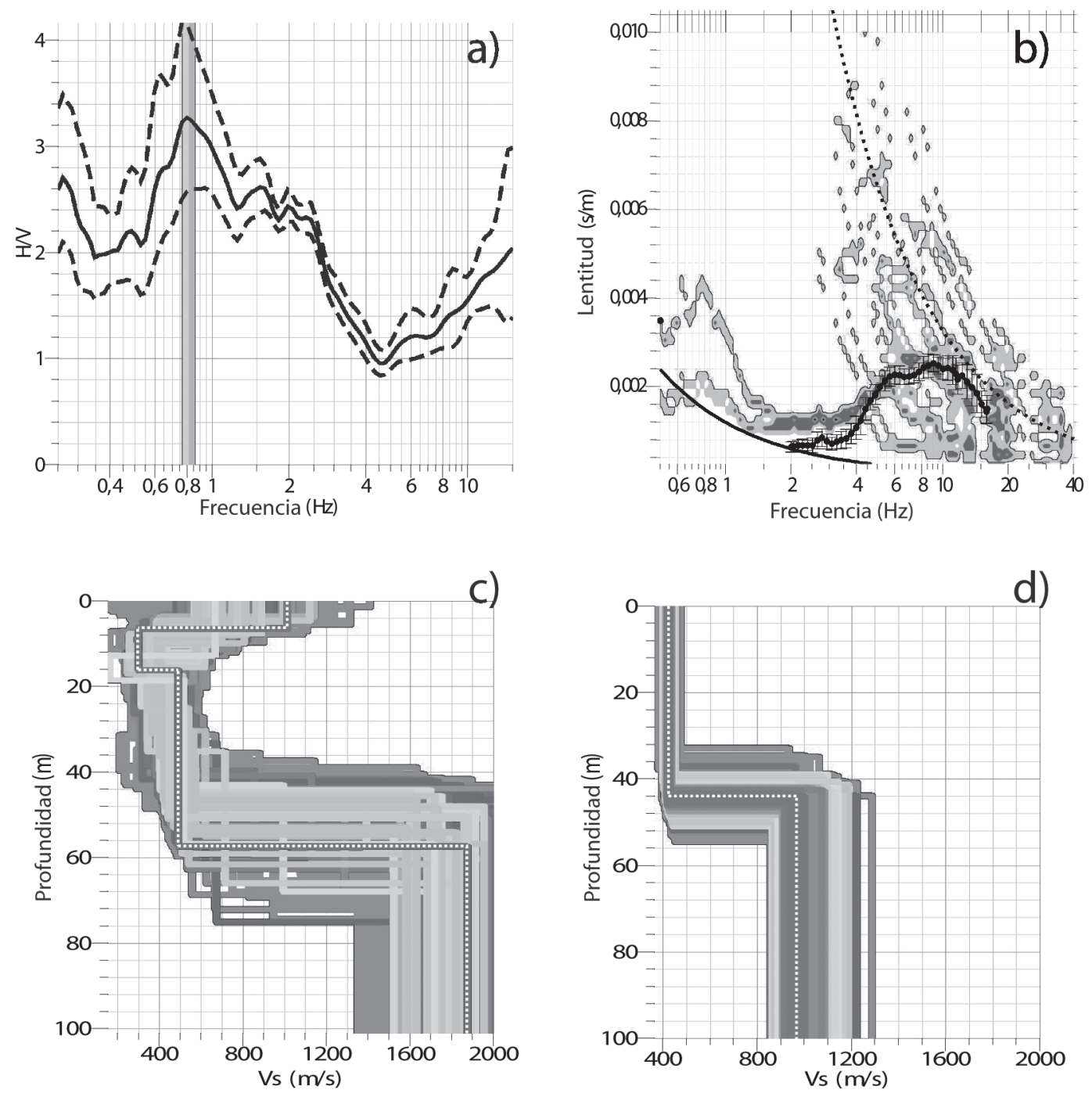

Fig. 14: Cancha para beisbol, TEC, Cartago (cerca de estación CTEC).

Plaza en el colegio St. Jude, Santa Ana (Fig. 17). $\mathrm{H} / \mathrm{V}$ se muestra muy plana hasta los $10 \mathrm{~Hz}$ aproximadamente, por lo que el medio parece ser más bien homogéneo y rígido. Esto lo confirma la curva de dispersión obtenida con FK, donde prácticamente no se observa curva, por lo que las ondas no manifiestan el fenómeno de variación de velocidad aparente respeto a la frecuencia, que es lo que significa la dispersión. Esto impide utilizar los métodos FK y SPAC para obtener modelos de Vs. Una limitación importante en este sitio es el poco espacio disponible para colocar los instrumentos. 

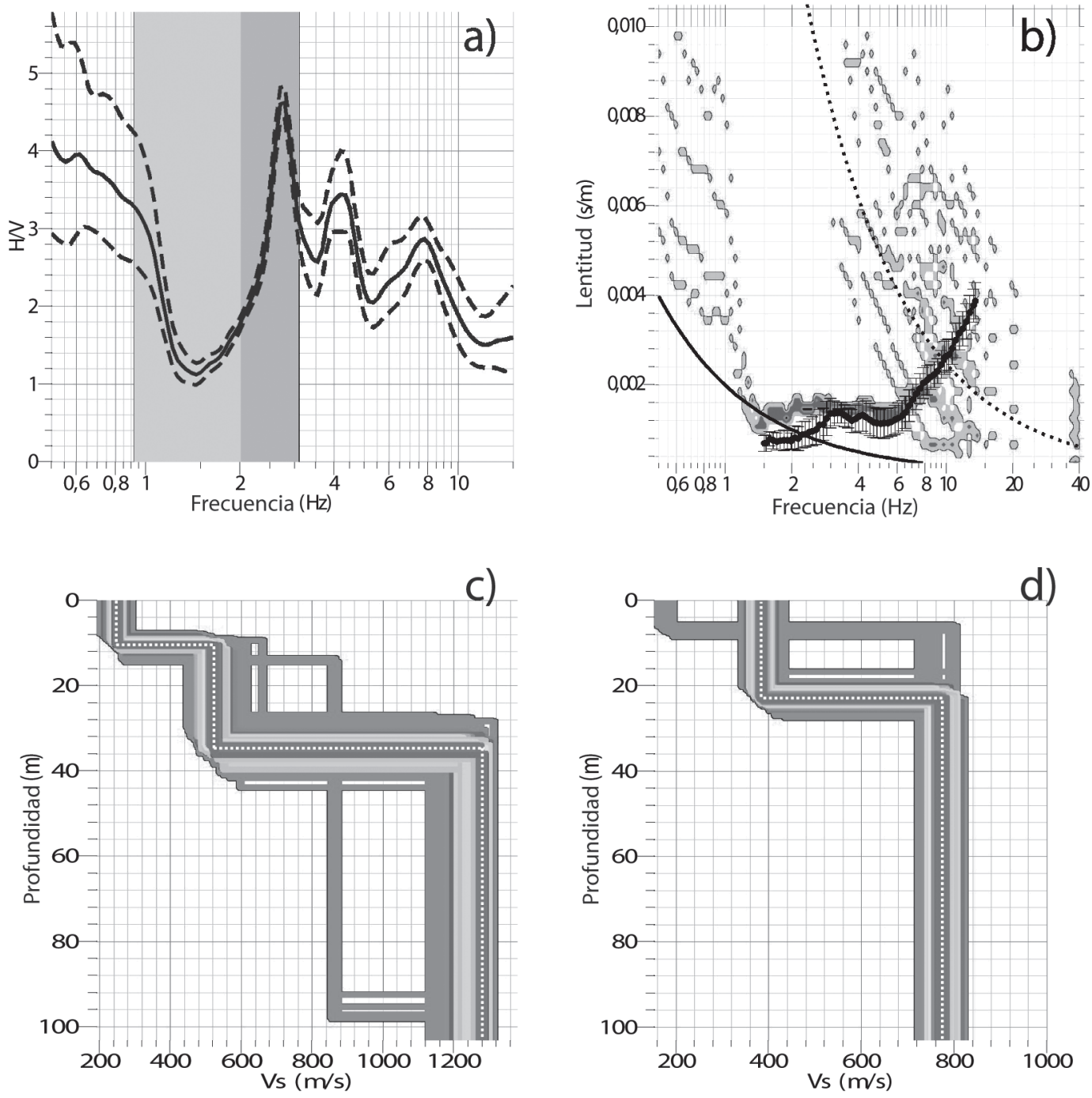

Fig. 15: Plaza en Hatillo, San José (cerca de estación SHTH).

Plaza al lado de la Facultad de Derecho, UCR (Fig. 18). Se definen bastante bien dos picos en $\mathrm{H} / \mathrm{V}$, siendo el de mayor amplitud el de alrededor de 3,6 Hz. Se evidencia una tendencia a subestimar la curva de dispersión en las bajas frecuencias por parte del método FK y hay

una cierta coincidencia en las tendencias de los métodos FK y SPAC. Solamente se obtuvo resultados claros usando FK, que es el que define aproximadamente con la misma dispersión dos capas con sus respectivos Vs y contrastes, así como el basamento. 

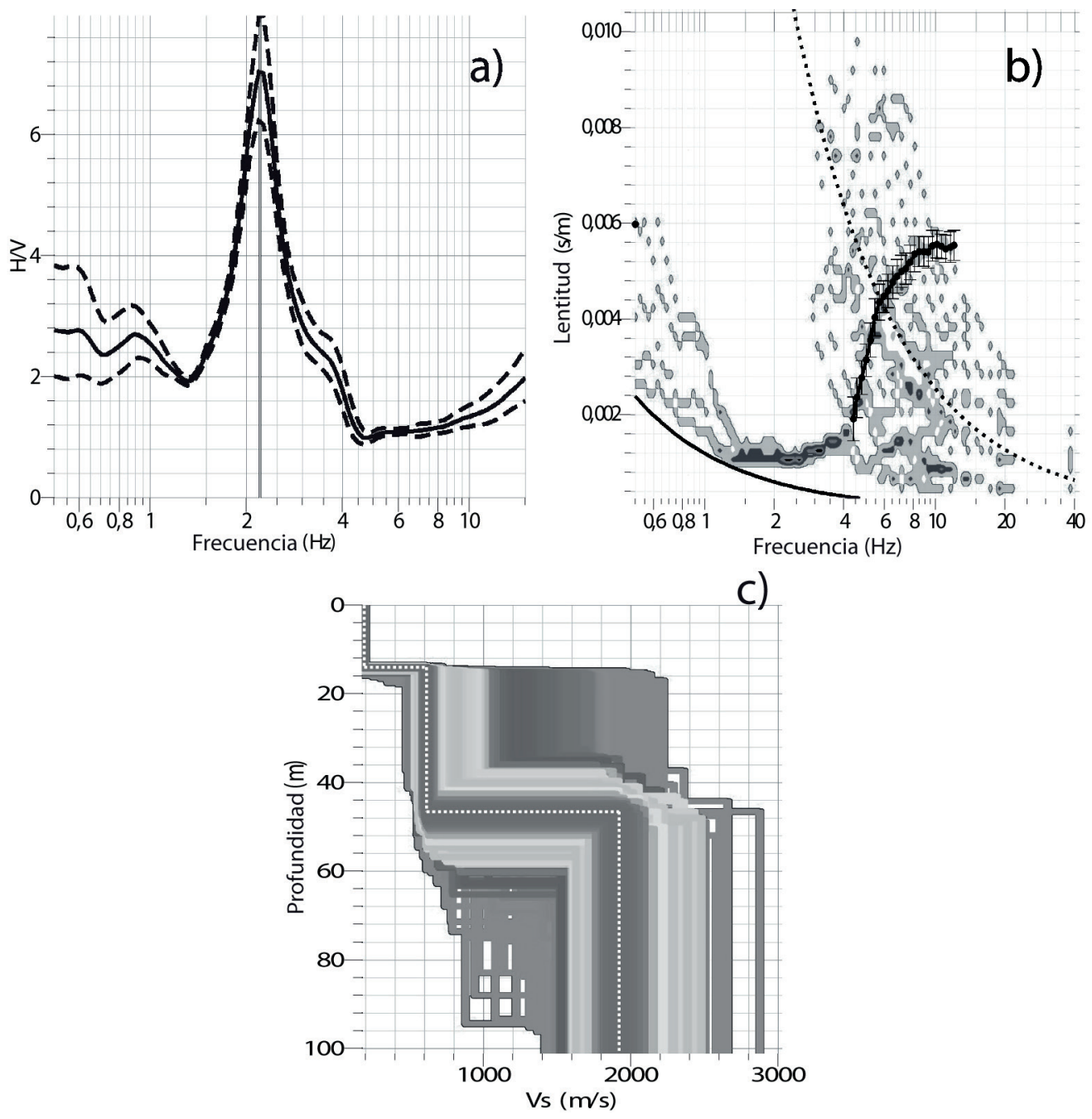

Fig. 16: Cancha de softball, La Sabana (cerca de estación SSBN).

Se trabajó en tres sitios adicionales a modo de pruebas y calibración de la metodología. No hay acelerógrafos cercanos a estos sitios.

Explanada al lado de Ingeniería Eléctrica, UCR (Fig. 19). Se evidencian dos picos en $\mathrm{H} / \mathrm{V}$, uno en $2,9 \mathrm{~Hz}$ y otro con amplitud inferior en 10 $\mathrm{Hz}$, que parecen estar claramente asociados al modelo de 2 capas mostrado por FK (no así por SPAC). Los mejores modelos generados por FK presentan poca dispersión y definen un primer contraste débil a $3,6 \mathrm{~m}$ y otro mayor a $20 \mathrm{~m}$ de profundidad. Las curvas de dispersión siguen tendencias semejantes pero de manera recurrente, a bajas frecuencias FK muestra menores valores de lentitud que SPAC. Por su parte, SPAC no genera modelos que permitan definir una primera capa superficial, pero si coincide bastante bien con la segunda capa definida por FK.

Estadio ecológico, instalaciones deportivas, Sabanilla, UCR (Fig. 20). Es clara la dispersión de los resultados de $\mathrm{H} / \mathrm{V}$, sobre todo en frecuencias bajas, debido probablemente a efectos del viento que generó vibraciones indeseadas en los geófonos o a problemas de estabilización del equipo, aún cuando estos fueron protegidos con cajas plásticas. Se logra evidenciar una frecuencia 


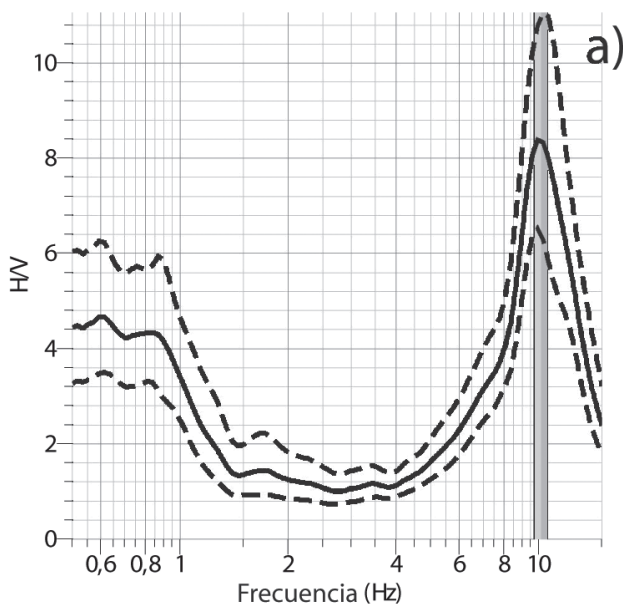

Fig. 17: Plaza en el colegio St. Jude, Santa Ana (cerca de estación SJUD).
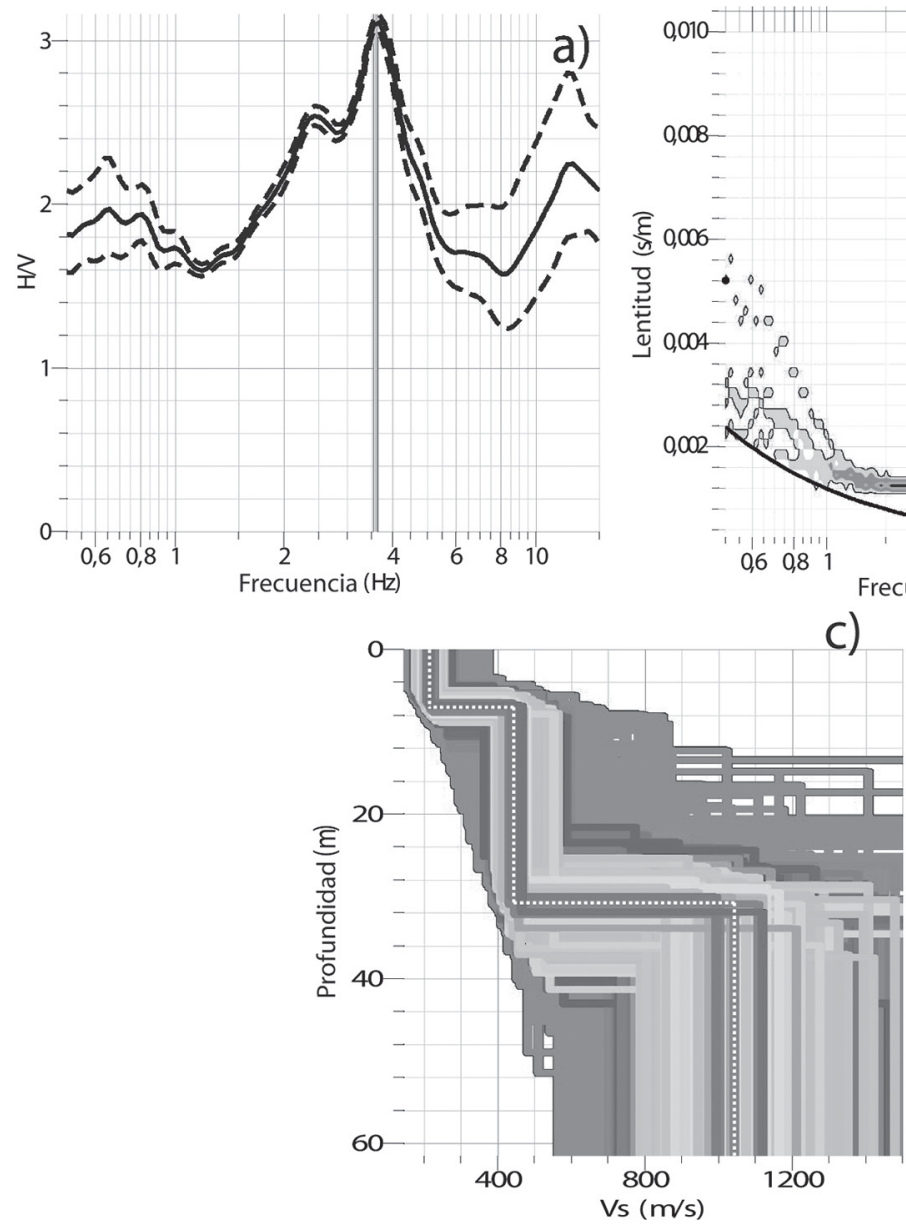

Fig. 18: Plaza al lado de la Facultad de Derecho, UCR (cerca de estación SLPF).

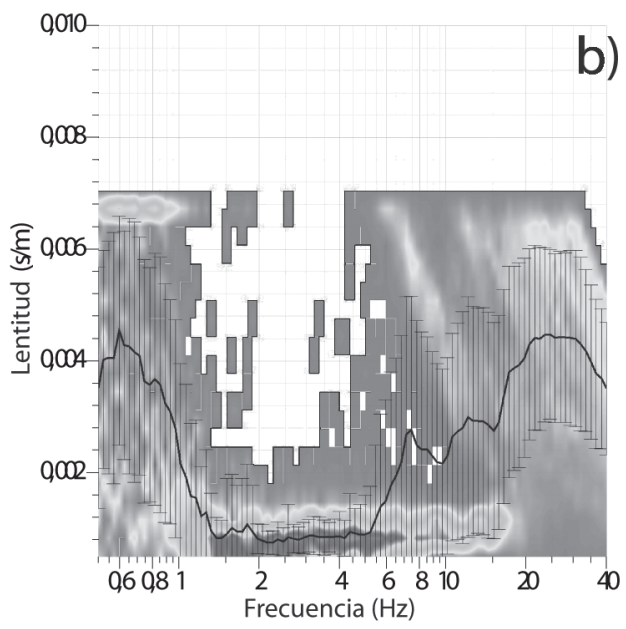

b)

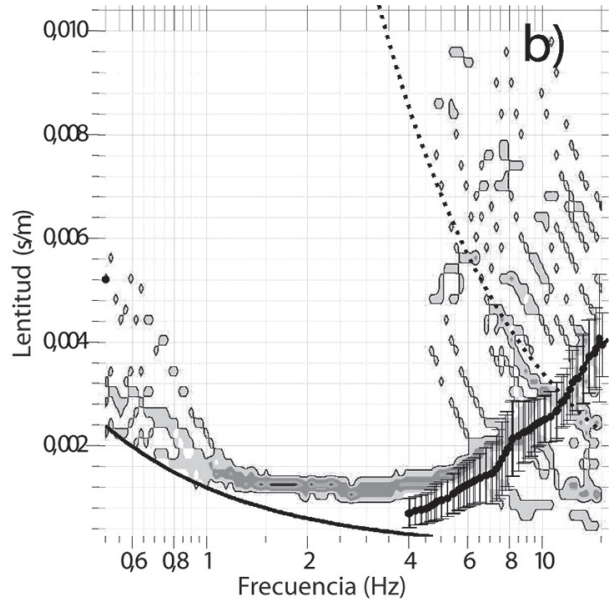

c) 


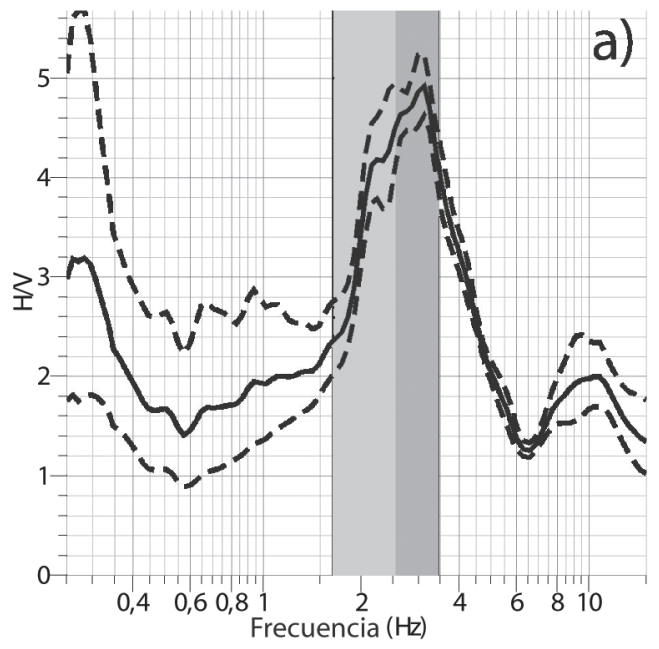

C)

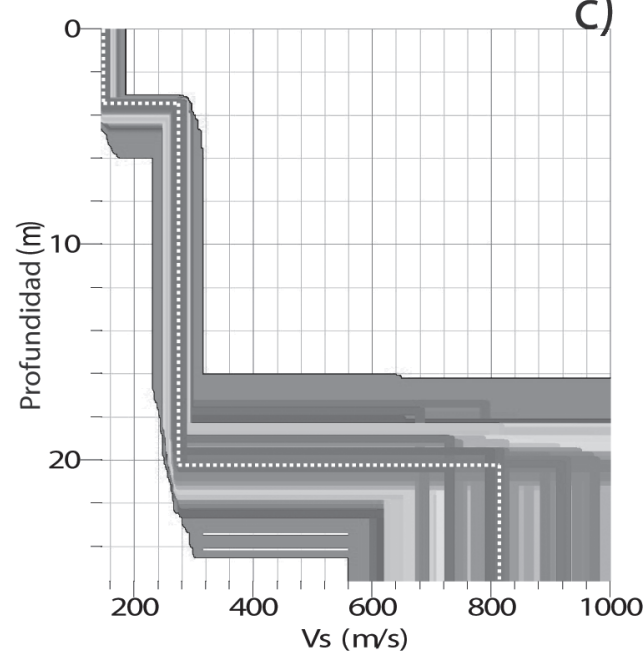

Fig. 19: Explanada al lado de Ingeniería Eléctrica, UCR.

fundamental en 2,1 Hz. La curva de dispersión no es tan clara según SPAC. Sin embargo, tanto FK como SPAC muestran modelos de una capa con Vs y espesor semejantes: $190 \mathrm{~m} / \mathrm{s}$ y $15 \mathrm{~m}$ aproximadamente.

Plaza Iglesias, Cartago (Fig. 21). Se define una frecuencia fundamental de $1 \mathrm{~Hz}$ según $\mathrm{H} / \mathrm{V}$, que sobre todo es claro en el eje de frecuencias
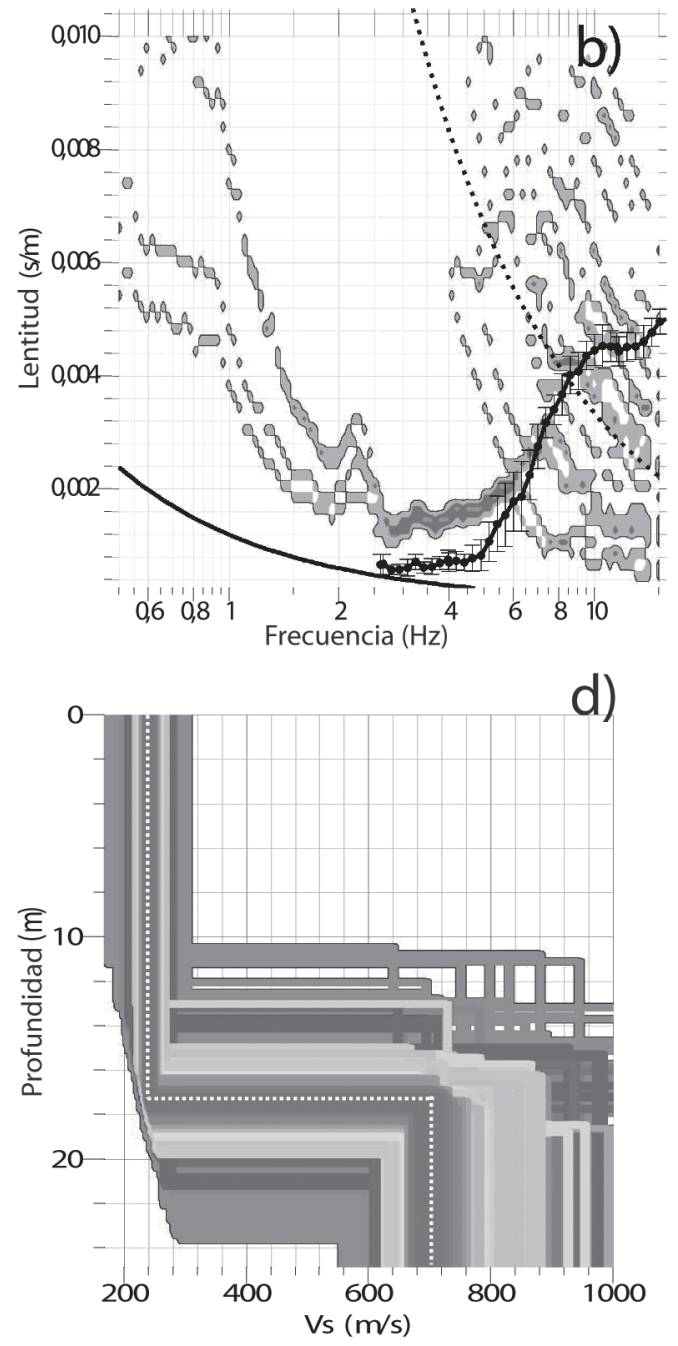

pero no tanto en el de las amplitudes. La curva de dispersión según el método FK cubre un rango más amplio de frecuencias que SPAC. Esto es concordante con los modelos generados por cada método, donde FK muestra mayor claridad de los resultados, aún cuando las Vs de la segunda capa y del basamento, así como la profundidad identificada para la base son bastante dispersas. 


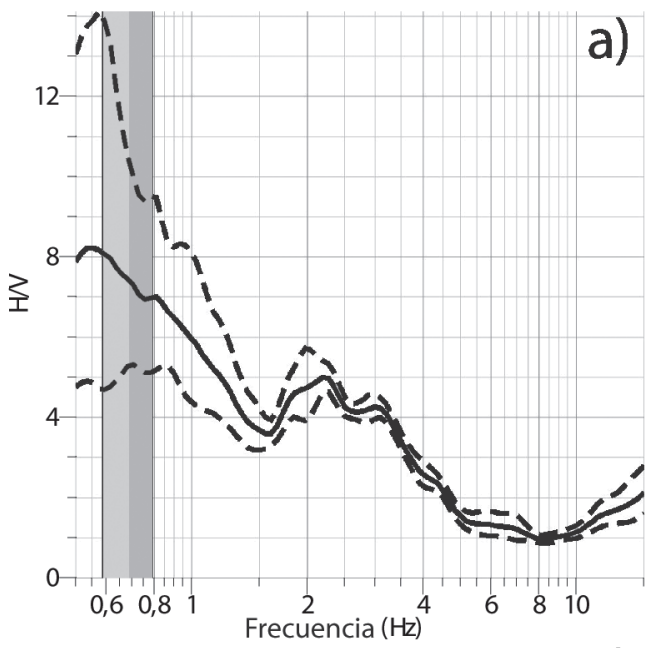

c)

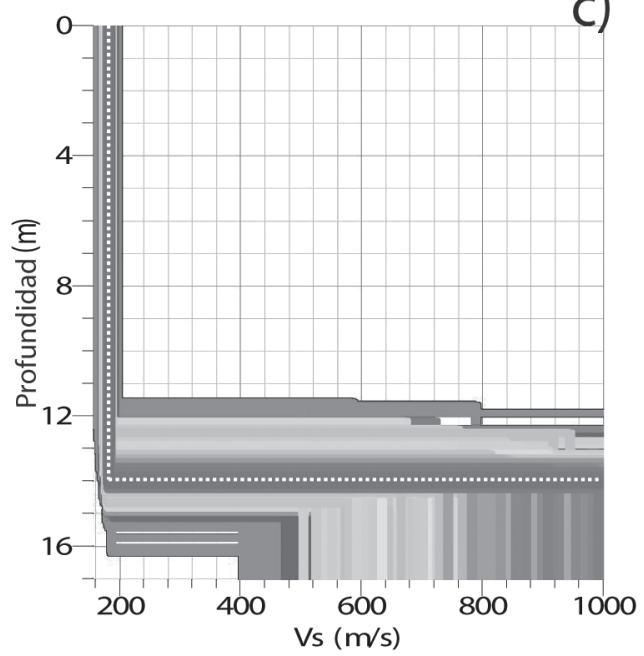

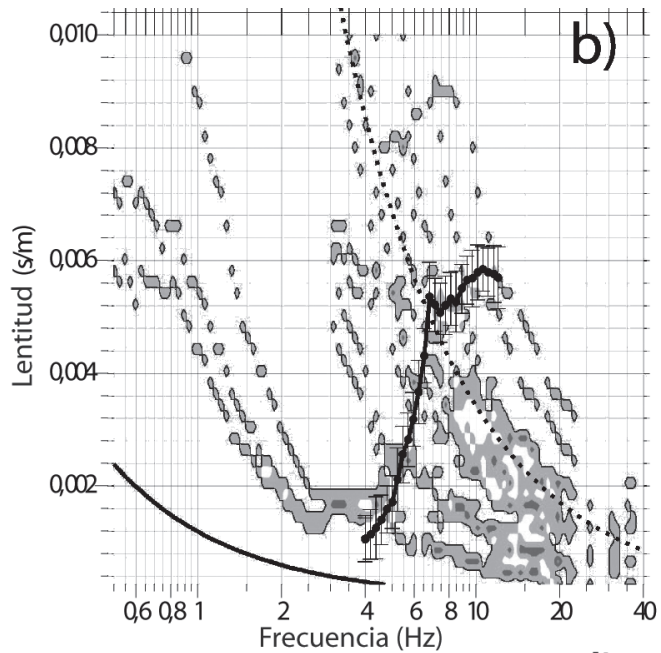

d)

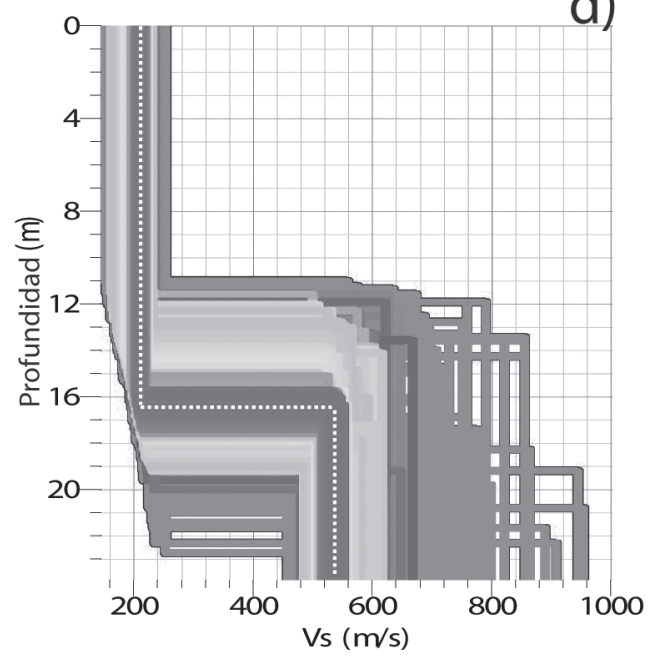

Fig. 20: Estadio ecológico, instalaciones deportivas, Sabanilla, UCR.

\section{DISCUSIÓN}

Los cuadros 4 al 7 presentan los valores de todos los parámetros estimados para cada sitio. Algunos de estos son: razón H/V para sismos (en cada estación acelerográfica), H/V para ruido en el sitio de la ubicación de los geófonos para la aplicación de la técnica de arreglos de sensores o arrays, distancia entre la estación y el sitio de ubicación de los geófonos, mejores modelos obtenidos con el uso de los métodos FK y SPAC, con su respectivo error o desajuste (misfit) y finalmente, la Vs30 para cada sitio con su respectiva clasificación de suelos.

La mayoría de los sitios donde fueron colocados los arreglos se ubican relativamente cerca de las estaciones cuyo suelo se desea caracterizar, 


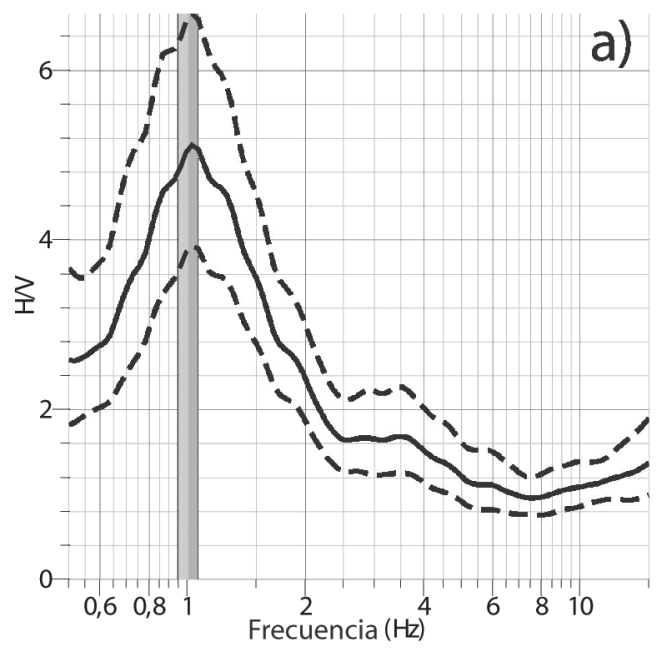

c)

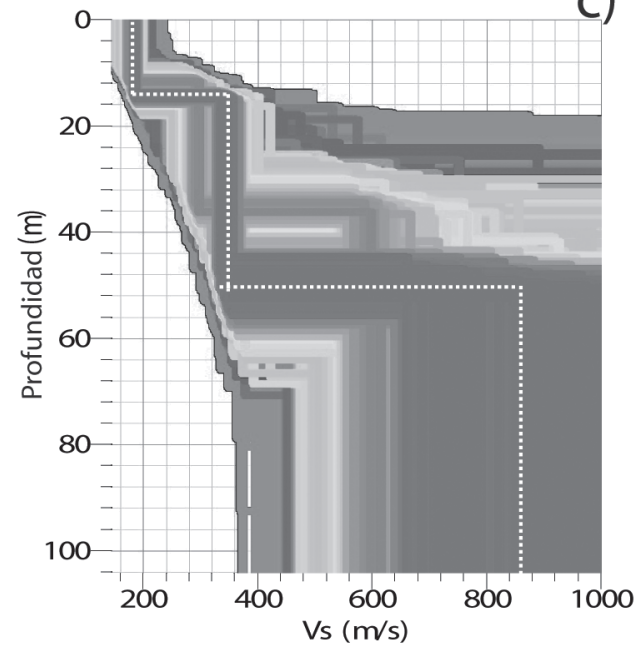

Fig. 21: Plaza Iglesias, Cartago.

siendo la más cercana CCRT (array a $65 \mathrm{~m}$ de la estación) y CPAR el más lejano (855 m entre la estación y el sitio). En este último caso, no hay un sitio cercano que sea adecuado para colocar los arreglos de sensores.

Respecto a los sitios que corresponden a estaciones (12 de 15 lugares estudiados), al tomar en cuenta que el periodo fundamental en el sitio de prueba y el de la estación deben asemejarse para asegurar que se está hablando de un mismo

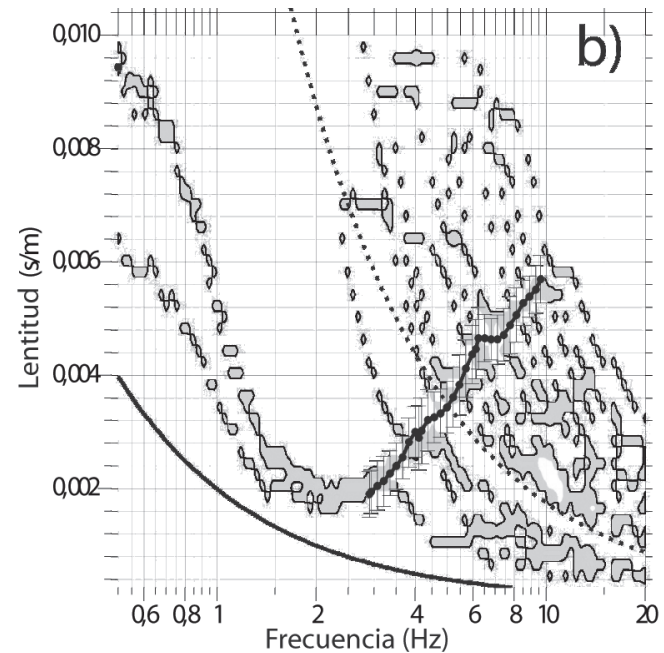

d)

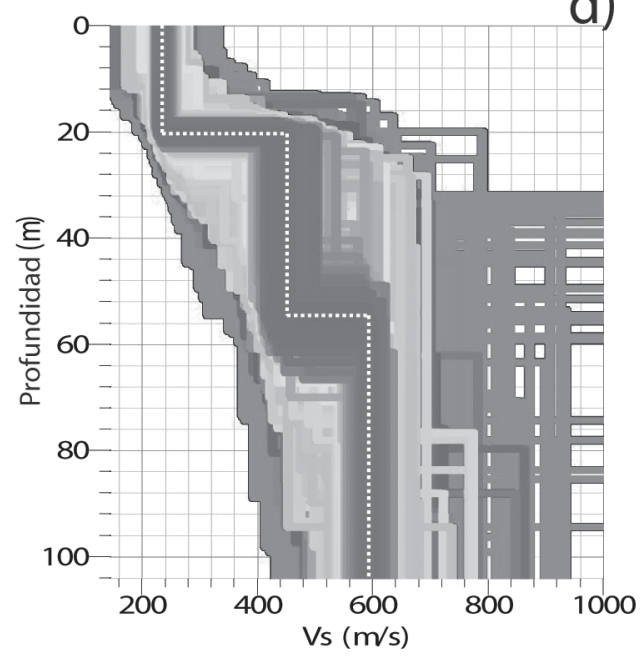

perfil de suelos en ambos sitios, fue necesario comparar los resultados de H/V usando sismos registrados en cada estación y ruido en los sitios donde se colocaron los sensores. Se obtuvieron los siguientes resultados:

- Muy similares (diferencia entre periodos menor o igual al 15\%). Sitios: AFBR, AGRE, ASRM, CCTR, SHTH, SSBN, SJUD, SLPF (8 de 12 sitios estudiados). 


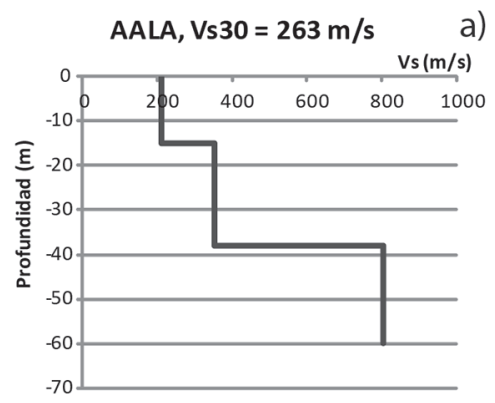

AFBR, $V s 30=283,5 \mathrm{~m} / \mathrm{s} \quad$ b)
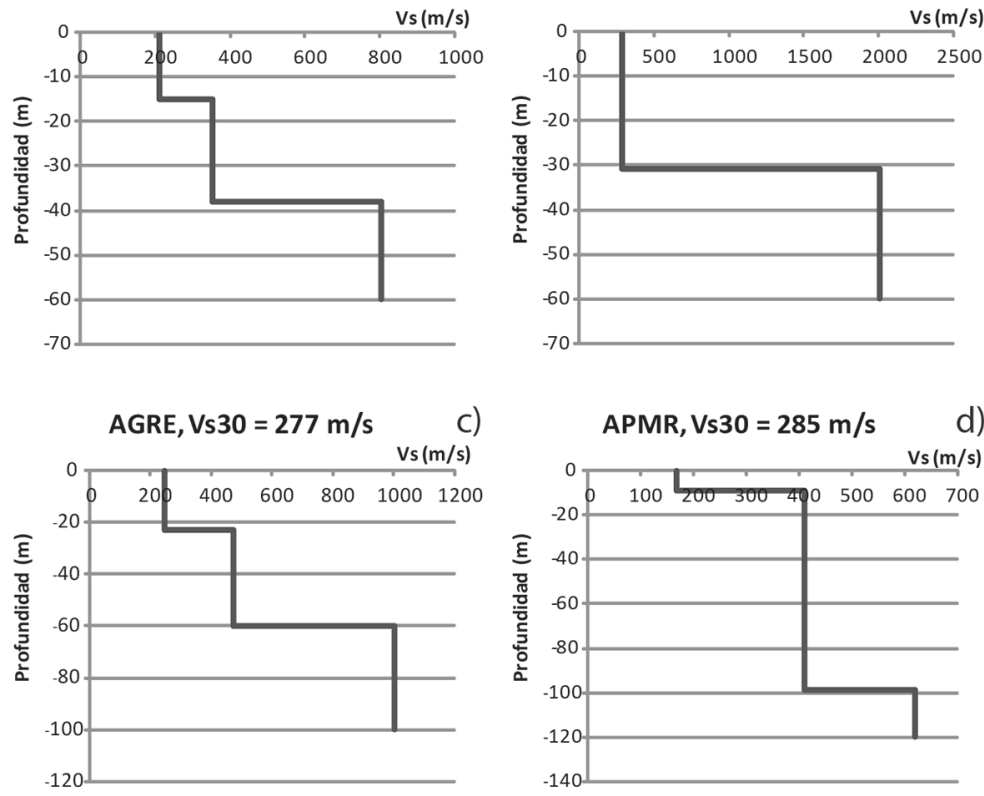

c)

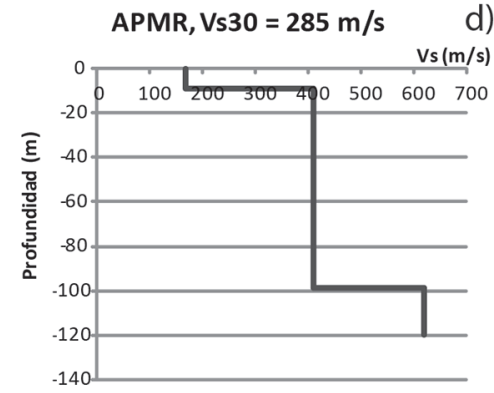

Fig. 22: Resultados finales en los sitios: AALA, AFBR, AGRE, APMR.
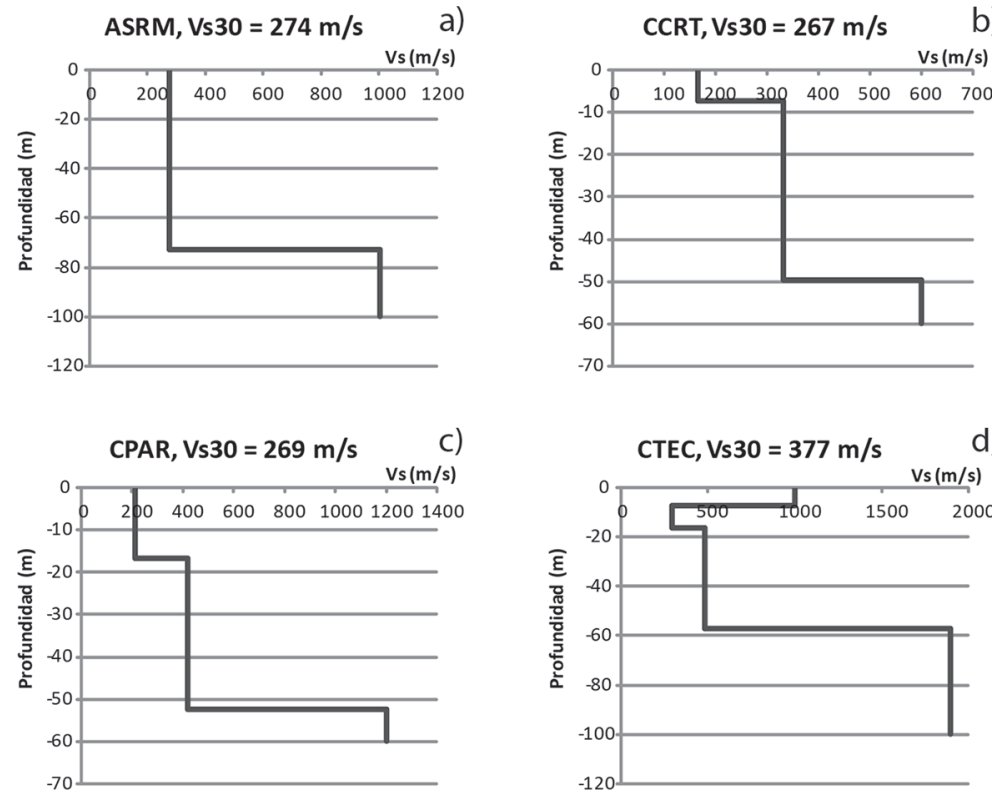

Fig. 23: Resultados finales en los sitios: ASRM, CCRT, CPAR y CTEC. 

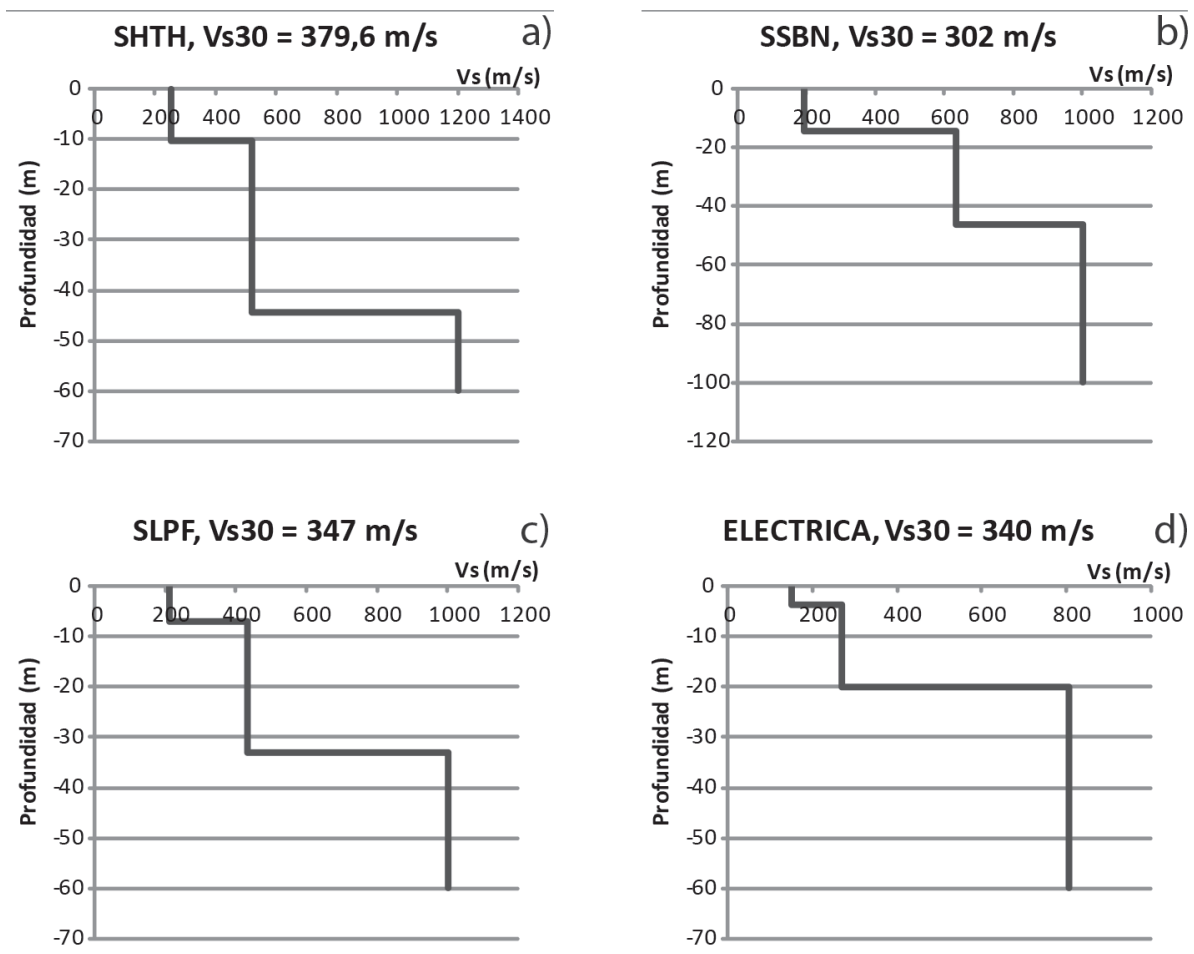

Fig. 24: Resultados finales en los sitios: SHTH, SSBN, SLPF, ELECTRICA.
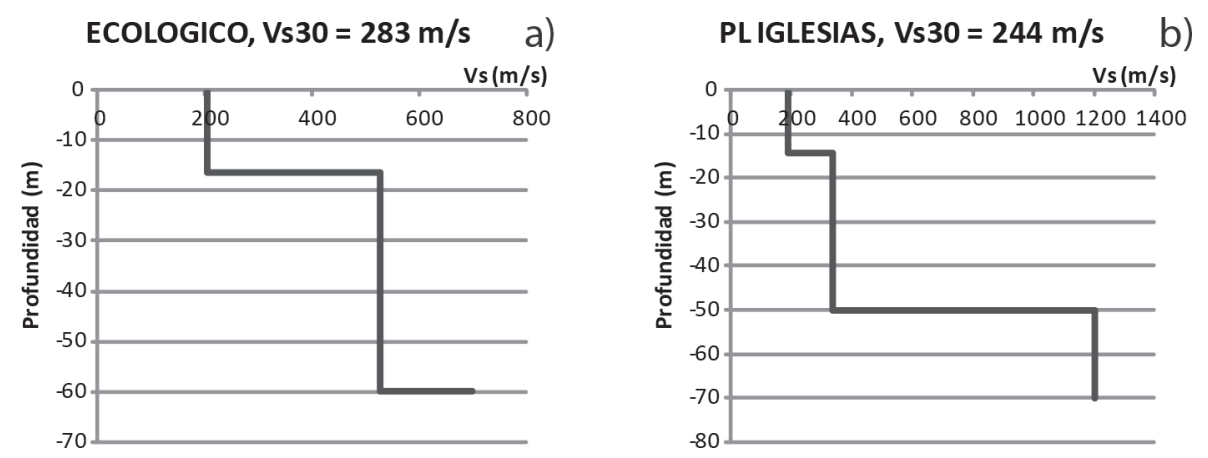

Fig. 25: Resultados finales en los sitios: ECOLÓGICO Y PL IGLESIAS.

- Aceptablemente similares (diferencia entre los periodos del $15 \%$ al $40 \%$ ). Sitios: AALA, APMR (2 de 12 sitios estudiados).

- Poco similares (diferencia entre los periodos superior al $40 \%$ ). Sitios: CPAR y CTEC. (2 de 12 sitios estudiados).

Se observa que en el $83 \%$ de los casos, los periodos en el sitio y en la estación coinciden muy bien o en forma aceptable, por lo que sí se pueden extrapolar los resultados del perfil de suelo que se obtenga en el sitio de prueba al de la estación.

Cabe señalar que tanto los sitios AALA como CPAR están bastante alejados de los lugares donde se colocaron los geófonos y no hay una muy buena concordancia entre los periodos fundamentales encontrados, por lo que será necesario aplicar alguna otra técnica geofísica 
Cuadro 4

Resumen de resultados en estaciones AALA, AFBR, AGRE y APMR

\begin{tabular}{|c|c|c|c|c|}
\hline Estación & AALA & AFBR & AGRE & APMR \\
\hline \multicolumn{5}{|l|}{$\mathrm{H} / \mathrm{V}$ con sismos } \\
\hline Periodo (s) & 0,54 & 0,55 & 0,8 & 1,5 \\
\hline Frecuencia $(\mathrm{Hz})$ & 1,85 & 1,82 & 1,25 & 0,67 \\
\hline Amplitud & 5 & 4 & 9,2 & 6 \\
\hline observaciones & $* * *$ & otro en 0,13 & $* * *$ & en 0,15 amp 6,8 \\
\hline Tipo de suelo ( To, Zhao) & S III & S III & S IV & S IV \\
\hline Distancia al sitio (m) & 600 & 200 & 210 & 520 \\
\hline \multirow[t]{2}{*}{ Sitio del array } & plaza & cancha futbol & Plaza futbol al & Estadio \\
\hline & Acosta & atrás & sur de UCR & Palmares \\
\hline \multicolumn{5}{|l|}{ H/V con ruido } \\
\hline Periodo & 0,71 & 0,48 & 0,77 & 2 \\
\hline Frecuencia & 1,42 & 2,07 & 1,3 & 0,5 \\
\hline Amplitud & 5,36 & 5,35 & 6,3 & 8,2 \\
\hline \multirow[t]{2}{*}{ Observ } & pico claro & otro pico en alg & disperso en & otro en $0,2 \mathrm{~s}$ \\
\hline & & $1,1 \mathrm{seg}$, amp 5 & baja frec viento & amp 5,0 \\
\hline Tipo de suelo ( To, Zhao) & S IV & S III & S IV & S IV \\
\hline \multicolumn{5}{|l|}{ Mejor modelo FK } \\
\hline Observ & 35 rang seguro & comb seguro & comb, amplio & aver amplio \\
\hline misfit & 0,23 & 0,17 & 0,2 & 0,76 \\
\hline $\mathrm{Vs} 1(\mathrm{~m} / \mathrm{s})$ & 210 & 277,46 & 245 & 160 \\
\hline H1 (m) & 15 & 26,42 & 22,7 & 9 \\
\hline Vs2 (m/s) & 352 & & 470 & 496 \\
\hline $\mathrm{H} 2(\mathrm{~m})$ & 23 & & 37,3 & 90 \\
\hline \multicolumn{5}{|l|}{$\mathrm{Vs} 3(\mathrm{~m} / \mathrm{s})$} \\
\hline \multicolumn{5}{|l|}{ H3 (m) } \\
\hline Vbasam (m/S) & 800 & 790 & 1000 & 1400 \\
\hline Periodo (s)1 & 0,55 & 0,38 & 0,69 & 0,95 \\
\hline Frecuencia & 1,82 & 2,63 & 1,45 & 1,05 \\
\hline
\end{tabular}

más cercana a la estación para caracterizar mejor esos sitios.

De acuerdo con la clasificación de suelos basada en el periodo fundamental del sitio (Zhao et al, 2006), leído de la razón $\mathrm{H} / \mathrm{V}$, se observan los siguientes resultados para todos los puntos donde se colocaron los arreglos:

- S IV: suelo blando a muy blando: AGRE, APMR, ASRM, CCRT, CPAR, Plaza Iglesias. (6 de 15 sitios $=40 \%$ ).
- S III: suelo medio a blando: AALA, AFBR, CTEC, SSBN, estadio ecológico. (5 de 15 sitios $=33 \%$ ).

- S II: suelo duro: SHTH, SLPF, SJUD, ingeniería eléctrica. ( 4 de 15 sitios $=27 \%$ ).

- S I: roca dura a roca meteorizada: no hay ningún sitio.

Una vez seleccionados los mejores métodos y modelos para cada sitio, se observa que aproximadamente la mitad se obtuvo con FK y la 
Cuadro 4 (continuación)

Resumen de resultados en estaciones AALA, AFBR, AGRE y APMR

\begin{tabular}{|c|c|c|c|c|}
\hline Estación & AALA & AFBR & AGRE & APMR \\
\hline Vs30 (m/s) & 263 & 300 & 277 & 304 \\
\hline Tipo de suelo & S 3 & S 3 & S 3 & S 3 \\
\hline \multicolumn{5}{|c|}{ Mejor modelo SPAC } \\
\hline Observ & comb & r 16,5 & 4 anillos comb & \\
\hline misfit & 0,3 & 0,35 & 0,39 & \\
\hline $\mathrm{Vs} 1(\mathrm{~m} / \mathrm{s})$ & 219 & 283,5 & 265 & 167 \\
\hline H1 (m) & 17,9 & 30,87 & 26,13 & 9 \\
\hline $\mathrm{Vs} 2(\mathrm{~m} / \mathrm{s})$ & 388 & & 448 & 409 \\
\hline H2 (m) & 29,7 & & 32,32 & 90 \\
\hline \multicolumn{5}{|l|}{ Vs3 (m/s) } \\
\hline \multicolumn{5}{|l|}{ H3 (m) } \\
\hline Vbasam $(\mathrm{m} / \mathrm{S})$ & 912 & 2000 & 737 & 620 \\
\hline Periodo (s) 1 & 0,63 & 0,44 & 0,68 & 1,1 \\
\hline Frecuencia & 1,58 & 2,27 & 1,47 & 0,91 \\
\hline Vs30 (m/s) & 265 & 283,5 & 280 & 285 \\
\hline Tipo de suelo & S 3 & S 3 & S 3 & S 3 \\
\hline
\end{tabular}

otra mitad con SPAC. En general, los modelos tienen a dar resultados más dispersos en las capas más profundas y sobre todo al definir la Vs del basamento. Esto puede deberse a la limitación en las aperturas de los arreglos utilizados en esta investigación, que permiten registrar adecuadamente el arribo de los frentes de onda que atraviesan las capas más superficiales, pero no son tan precisos con los registros que provienen de capas profundas.

Los errores o misfit generados en los modelos van de 0,2 en AGRE (menor error) a 0,79 en SSBN (mayor de los errores observados), siendo la mayoría alrededor de 0,4 . Solamente para SJUD no se logró generar ningún modelo ya que corresponde a un sitio aparentemente muy homogéneo, sin contrastes importantes en profundidad que hayan podido ser identificados por el arreglo de sensores (no se observan picos en $\mathrm{H} / \mathrm{V}$ ).

Los valores de Vs30 obtenidos van de 379,6 $\mathrm{m} / \mathrm{s}$ en SHTH (que es la mayor de todas) a $244 \mathrm{~m} / \mathrm{s}$ en Plaza Iglesias de Cartago, que es la Vs30 menor. Este rango parece indicar que los suelos que predominan son $\mathrm{S}_{3}$ según la clasificación dada por el CSCR-2010, ya que esta clase corresponde a un rango de Vs30 entre 350 y $180 \mathrm{~m} / \mathrm{s}$.

Hay algunos casos como AALA, APMR, CCRT, CPAR y CTEC en los que el periodo obtenido con $\mathrm{H} / \mathrm{V}$ y el generado a partir de modelos difieren. Este último se calculó según la siguiente ecuación (Kramer, 1996):

$$
T_{0}=4 * \sum_{i=1}^{n} \frac{h_{i}}{V s_{i}}
$$

Siendo $\mathrm{T}_{0}$ el periodo fundamental o característico del sitio, hi la profundidad del estrato "i" y Vsi la velocidad de onda cortante del estrato "i".

La situación planteada en el párrafo anterior se debe a que los arreglos realizados en esos sitios tienen poca apertura (poco diámetro debido a limitaciones físicas del espacio) y no logran identificar capas más profundas del perfil de suelo, por lo que el periodo fundamental $\left(\mathrm{T}_{0}\right)$ obtenido con $\mathrm{H} / \mathrm{V}$ es mayor que el periodo obtenido con el perfil. En otras palabras, el del perfil representa 
Cuadro 5

Resumen de resultados en estaciones ASRM, CCRT, CPAR y CTEC

\begin{tabular}{|c|c|c|c|c|}
\hline Estación & ASRM & CCRT & CPAR & CTEC \\
\hline \multicolumn{5}{|l|}{ H/V con sismos } \\
\hline Periodo (s) & 1,1 & 1,3 & 0,5 & 0,45 \\
\hline Frecuencia (Hz) & 0,91 & 0,77 & 2 & 2,22 \\
\hline Amplitud & 8 & 4 & 7,5 & 5 \\
\hline observaciones & $* * *$ & amplio & otro en $0,9 \mathrm{~s}$ & $* * *$ \\
\hline Tipo de suelo ( To, Zhao) & S IV & S IV & S III & S III \\
\hline Distancia al sitio (m) & 340 & 65 & 855 & 170 \\
\hline \multirow{2}{*}{ Sitio del array } & cancha futbol & plaza al lado & cancha futbol & Cancha beisbol \\
\hline & con pista & biblioteca & cerca L Doña Ana & Tecnologico \\
\hline \multicolumn{5}{|l|}{$\mathrm{H} / \mathrm{V}$ con ruido } \\
\hline Periodo & 1,05 & 1,11 & 0,91 a 1,1 & 1,26 a 1,16 \\
\hline Frecuencia & 0,95 & 0,9 & 1,1 a 0,91 & 0,79 a 0,86 \\
\hline Amplitud & 8,8 & 5 & 5,5 & 3,3 \\
\hline Observ & $* * *$ & dispersión & muy disperso, $\mathrm{T}_{0}$ & disperso, otro \\
\hline & & & variable & pico $0,11 \mathrm{~s}, 2,7 \mathrm{a}$ \\
\hline Tipo de suelo ( To, Zhao) & S IV & S IV & S IV & S IV \\
\hline \multicolumn{5}{|l|}{ Mejor modelo FK } \\
\hline Observ & & amplio combin & curv comb amplio & aver amplio \\
\hline misfit & 0,49 & 0,57 & 0,35 & 0,75 \\
\hline $\mathrm{Vs} 1(\mathrm{~m} / \mathrm{s})$ & 279,34 & 203 & 210 & 1002 \\
\hline $\mathrm{H} 1$ (m) & 52 & 10 & 16,67 & 7 \\
\hline $\mathrm{Vs} 2(\mathrm{~m} / \mathrm{s})$ & & 352 & 416 & 296 \\
\hline $\mathrm{H} 2(\mathrm{~m})$ & & 39 & 35,76 & 9 \\
\hline $\mathrm{Vs} 3(\mathrm{~m} / \mathrm{s})$ & & & & 487 \\
\hline H3 (m) & & & & 41 \\
\hline Vbasam $(\mathrm{m} / \mathrm{S})$ & $<2000$ & $>1000$ & $>1200$ & 1896 \\
\hline Periodo (s)1 & 0,74 & 0,64 & 0,66 & 0,486 \\
\hline Frecuencia & 1,35 & 1,56 & 1,52 & 2,06 \\
\hline
\end{tabular}

solo una parte del $\mathrm{T}_{0}$ real en el sitio, al no poderse obtener información de capas más profundas.

El sitio en el que se manifiestan más inconsistencias en la clasificación de suelos (diferencias sustanciales entre el método de Zhao et al., 2006, basado en $\mathrm{T}_{0}$ y el criterio de Vs30 según el CSCR2010) es CTEC (S IV con un método y $S_{2}$ con otro), debido probablemente a irregularidades del sitio. Será necesario aplicar nuevamente la técnica de arrays (FK, SPAC o bien el análisis múltiple de ondas superficiales MASW) en un sitio cercano para corroborar resultados, posiblemente donde se ubica la pista de atletismo de ese recinto.

En general, se observa que los sitios clasificados según el método de arrays predominan los tipos $\mathrm{S}_{3}$ (según CSCR-2010) y usando H/V (identificación de $\mathrm{T}_{0} \mathrm{y}$ aplicando los criterios según Zhao et al, 2006), predomina S IV. 
Cuadro 5 (continuación)

Resumen de resultados en estaciones ASRM, CCRT, CPAR y CTEC

\begin{tabular}{|c|c|c|c|c|}
\hline Estación & ASRM & CCRT & CPAR & CTEC \\
\hline Vs30 (m/s) & 279 & 290 & 269 & 377 \\
\hline Tipo de suelo & S 3 & S 3 & S 3 & S 2 \\
\hline \multicolumn{5}{|c|}{ Mejor modelo SPAC } \\
\hline Observ & 7 anillos comb & comb & anillos combi (6) & todos aniill 2 capas \\
\hline misfit & 0,53 & 0,63 & 0,38 & 0,52 \\
\hline $\mathrm{Vs} 1(\mathrm{~m} / \mathrm{s})$ & 274,32 & 166 & 218,5 & 425 \\
\hline H1 (m) & 72,86 & 7,3 & 18 & 44 \\
\hline $\mathrm{Vs} 2(\mathrm{~m} / \mathrm{s})$ & & 332 & 378 & \\
\hline H2 (m) & & 42,47 & 24,16 & \\
\hline \multicolumn{5}{|l|}{ Vs3 (m/s) } \\
\hline \multicolumn{5}{|l|}{ H3 (m) } \\
\hline Vbasam $(\mathrm{m} / \mathrm{S})$ & 1000 & 600 & 650 & 1009 \\
\hline Periodo (s)1 & 1,06 & 0,69 & 0,586 & 0,41 \\
\hline Frecuencia & 0,94 & 1,45 & 1,71 & 2,4 \\
\hline Vs30 (m/s) & 274 & 267 & 263 & 425 \\
\hline Tipo de suelo & S 3 & S 3 & S 3 & S 2 \\
\hline
\end{tabular}

Lo anterior se debe a que los límites de Vs30 definidos por ambos criterios difieren ligeramente, pero en general se está hablando del mismo tipo de suelo en uno y en otro caso.

Finalmente, los gráficos de las figuras 22 a 25 muestran los perfiles de suelo para cada sitio con su respectiva Vs30, que representa el resultado final de este estudio.

1 El periodo según FK y SPAC de los cuadros 4 al 7 se calculó con la ecuación (2).

\section{CONCLUSIONES}

En total se clasificaron los suelos en 15 sitios: 12 de estaciones acelerográficas del Laboratorio de Ingeniería Sísmica y tres localidades que sirvieron para realizar pruebas preliminares al equipo adquirido.

En cada uno de ellos, se obtuvo el periodo fundamental con base en la técnica $\mathrm{H} / \mathrm{V}$ a partir de ruido y también con el uso de sismos. Se aplicaron también los métodos FK y SPAC para obtener Vs30.

Con respecto a la razón $\mathrm{H} / \mathrm{V}$, los sitios donde se define claramente un único pico (un único contraste evidente entre capas) fueron: Alajuela Plaza Acosta, Alajuela Fabio Baudrit, San Ramón UCR, cancha de softball de La Sabana y plaza Iglesias de Cartago. Los sitios donde se observan dos o más picos correspondientes probablemente a dos o más contrastes son: estadio de Palmares, plaza en Hatillo, plaza al lado de Derecho UCR e ingeniería eléctrica UCR. En los demás sitios, la identificación de $\mathrm{H} / \mathrm{V}$ es más confusa, como es el caso de la plaza del colegio St. Jude, donde se observa una razón $\mathrm{H} / \mathrm{V}$ casi plana en todo el rango de frecuencias y con una amplitud cercana a la unidad, asimismo una curva de dispersión casi horizontal, lo que permite concluir acerca de la presencia de un medio muy homogéneo y sin contrastes, por lo tanto sin dispersión.

Debido a que en esta investigación se pretendió clasificar los suelos bajo las estaciones acelerográficas y a que no siempre fue posible encontrar sitios abiertos para colocar los arreglos de sensores, se tuvo casos como CCRT donde la separación entre la estación y el sitio de prueba es de solo $65 \mathrm{~m}$, pero otros como CPAR, donde 
Cuadro 6

Resumen de resultados en estaciones SHTH, SSBN, SJUD y SLPF

\begin{tabular}{|c|c|c|c|c|}
\hline Estación & SHTH & SSBN & SJUD & SLPF \\
\hline \multicolumn{5}{|l|}{$\mathrm{H} / \mathrm{V}$ con sismos } \\
\hline Periodo (s) & 0,3 a 0,5 & 0,48 & plano & 0,31 \\
\hline Frecuencia (Hz) & 3,33 a 2 & 2,08 & plano & 3,23 \\
\hline Amplitud & 4 & 7 & plano & 6,2 \\
\hline observaciones & varios picos & $* * *$ & $* * *$ & $* * *$ \\
\hline Tipo de suelo ( To, Zhao) & S II - S III & S III & S I - S II & S II \\
\hline Distancia al sitio (m) & 450 & 250 & 110 & 152 \\
\hline Sitio del array & $\begin{array}{c}\text { Plaza Esc. Pac Fer } \\
\text { Hatillo }\end{array}$ & $\begin{array}{c}\text { Sabana } \\
\text { canchas futbol }\end{array}$ & $\begin{array}{l}\text { Cancha Futbol } \\
\text { Colegio St. Jude }\end{array}$ & $\begin{array}{l}\text { cancha futbol } \\
\text { Derecho }\end{array}$ \\
\hline \multicolumn{5}{|l|}{$\mathrm{H} / \mathrm{V}$ con ruido } \\
\hline Periodo & 0,37 & 0,47 & 0,1 & 0,28 \\
\hline Frecuencia & 2,72 & 2,12 & 10,4 & 3,5 a 3,6 \\
\hline Amplitud & 5 & 10 & 11,5 & 3,1 \\
\hline Observ & $\begin{array}{c}\text { otros picos en } \\
0,24 \text { y } 0,13 \mathrm{~s}\end{array}$ & pico claro & $\begin{array}{c}\text { plano hasta } \\
0,1 \mathrm{~s}\end{array}$ & $\begin{array}{c}\text { otro pico en } \\
0,43 \text { seg amp } 2,5\end{array}$ \\
\hline Tipo de suelo ( To, Zhao) & S II & S III & S I - S II & S II \\
\hline \multicolumn{5}{|l|}{ Mejor modelo FK } \\
\hline Observ & 3 cap prom ampl & 3 cap aver amplio & plano, no hay & \\
\hline misfit & 0,64 & 0,79 & dispersion & \\
\hline Vs1 (m/s) & 250 & 194 & & 212,35 \\
\hline H1 (m) & 10,2 & 14,5 & & 7 \\
\hline Vs2 (m/s) & 518 & 632 & & 431 \\
\hline $\mathrm{H} 2(\mathrm{~m})$ & 34,29 & 31,5 & & 26 \\
\hline \multicolumn{5}{|l|}{ Vs3 (m/s) } \\
\hline \multicolumn{5}{|l|}{$\mathrm{H} 3(\mathrm{~m})$} \\
\hline Vbasam (m/S) & 1200 & $<1000$ & & $<1000$ \\
\hline Periodo (s) 1 & 0,35 & 0,5 & & 0,317 \\
\hline Frecuencia & 2,86 & 2 & & 3,15 \\
\hline $\mathrm{Vs} 30(\mathrm{~m} / \mathrm{s})$ & 379,6 & 302 & & 347 \\
\hline Tipo de suelo & $\mathrm{S} 2$ & S 3 & & S 3 \\
\hline
\end{tabular}

la estación está alejada $855 \mathrm{~m}$ del espacio donde fueron colocados los sensores.

Tomando en cuenta lo anterior, hay principalmente tres sitios donde no hay una buena correspondencia entre el periodo fundamental obtenido a partir de $\mathrm{H} / \mathrm{V}$ con sismos registrados en la estación y $\mathrm{H} / \mathrm{V}$ obtenida a partir de ruido en los sitios de prueba. Estos sitios son: CPAR y AALA, que están muy alejados de los sitios de pruebas y CTEC, que representa un sitios con suelos muy variables en distancias muy cortas. En este lugar será necesario aplicar alguna técnica más cercana a la estación, que implique poca disponibilidad de espacio para su aplicación, como es el caso de MASW (Multichannel Analysis of Seismic Waves). 
Cuadro 6 (continuación)

Resumen de resultados en estaciones SHTH, SSBN, SJUD y SLPF

\begin{tabular}{ccccc}
\hline Estación & SHTH & SSBN & SJUD & SLPF \\
\hline Mejor modelo SPAC & & & & se aleja mucho \\
Observ & 2 capas 5 anillos & con anillos $(6)$ & plano, no hay & de H/V \\
misfit & 0,51 & 0,62 & 272 & \\
Vs1 (m/s) & 384 & 26,6 & \\
H1 (m) & 23 & & \\
Vs2 (m/s) & & & \\
H2 $(\mathrm{m})$ & & & \\
Vs3 $(\mathrm{m} / \mathrm{s})$ & & & \\
H3 $(\mathrm{m})$ & & & \\
Vbasam $(\mathrm{m} / \mathrm{S})$ & 770 & 0,39 & \\
Periodo $(\mathrm{s}) 1$ & 0,24 & 2,56 & \\
Frecuencia & 4,18 & 296 & \\
Vs30 $(\mathrm{m} / \mathrm{s})$ & 434 & S 3 & \\
Tipo de suelo & S 2 & & \\
\hline
\end{tabular}

Respecto a la clasificación de los sitios basada en el periodo fundamental (Zhao, et al., 2006), un $40 \%$ obtuvo una clasificación como $\mathrm{S}$ IV (blando), un 33\% como S III (medio), un $27 \%$ como S II (firme) y un $0 \%$ como roca.

Para la aplicación de las técnicas FK y SPAC, de los 15 sitios en estudio, en 12 se utilizaron 8 sensores, en 4 sitios fueron colocados 7 geófonos y en dos lugares se usaron 6. El sitio con mayor apertura del arreglo o mayor distancia entre geófonos fue $\mathrm{La}$ Sabana (92 m de diámetro mayor) y los de menor fueron CCRT, plaza de Derecho UCR e ingeniería eléctrica UCR (50 m de diámetro menor).

En todos los casos, las tendencias de las curvas de dispersión generadas por FK y SPAC son semejantes, observándose valores de lentitud menores correspondientes a frecuencias bajas según el método FK.

Aún cuando los casos de análisis considerados son solamente 15 , se observa una tendencia de que los modelos en profundidad sean más dispersos al aplicar FK que SPAC.

Los valores de Vs30 obtenidos con los mejores modelos generados por FK y SPAC van de $244 \mathrm{~m} / \mathrm{s}$ en plaza Iglesias de Cartago (menor Vs30 de todos) a 379,6 m/s en SHTH (mayor de todas). Este rango parece indicar que los suelos que predominan son tipo S3 según el CSCR-2010, ya que esta clase corresponde a un rango de Vs30 entre 180 y $350 \mathrm{~m} / \mathrm{s}$.

De acuerdo con los criterios del CSCR2010, los suelos en los sitios de estudio se clasifican de acuerdo al parámetro Vs30 de la siguiente manera:

$$
\mathrm{S}_{4}(\mathrm{Vs} 30<180 \mathrm{~m} / \mathrm{s}) \text { : ningún sitio, } 0 \%
$$

$\mathrm{S}_{3}(180 \mathrm{~m} / \mathrm{s}<\mathrm{Vs} 30<350 \mathrm{~m} / \mathrm{s}):$ AALA, AFBR, AGRE, APMR, ASRM, CCRT, CPAR, SSBN, ecológico UCR, pl. Iglesias Cartago (10 sitios, 67\%). 


\section{Cuadro 7}

Resumen de resultados en sitios: Ing. Eléctrica, Estadio ecológico y Pl. Iglesias

\begin{tabular}{|c|c|c|c|}
\hline Sitio del array & Ing. Electrica (UCR) & Estadio ecológico (UCR) & Plaza Iglesias (Cartago) \\
\hline \multicolumn{4}{|l|}{$\mathrm{H} / \mathrm{V}$ con ruido } \\
\hline Periodo & 0,34 & 0,48 & 1 \\
\hline Frecuencia & 2,9 & 2,1 & 1 \\
\hline Amplitud & 5,1 & 5 & 5,1 \\
\hline Observ & bien definido & $* * *$ & bien definido \\
\hline Tipo de suelo ( To, Zhao) & S II & S III & S IV \\
\hline \multicolumn{4}{|l|}{ Mejor modelo FK } \\
\hline Observ & 2 capas & 1 capa 16,6 seg & rango seguro \\
\hline misfit & 0,56 & 0,42 & 0,27 \\
\hline $\mathrm{Vs} 1(\mathrm{~m} / \mathrm{s})$ & 151 & 182 & 186,68 \\
\hline H1 (m) & 3,6 & 13,84 & 14,11 \\
\hline Vs2 (m/s) & 270 & & 336 \\
\hline $\mathrm{H} 2(\mathrm{~m})$ & 16.4 & & 35,89 \\
\hline \multicolumn{4}{|l|}{ Vs3 (m/s) } \\
\hline \multicolumn{4}{|l|}{ H3 (m) } \\
\hline $\operatorname{Vbasam}(\mathrm{m} / \mathrm{S})$ & 804,5 & 1000 & 1200 \\
\hline Periodo (s)1 & 0,345 & 0,3 & 0,9 \\
\hline Frecuencia & 2,90 & 3,33 & 1,11 \\
\hline Vs30 (m/s) & 340 & 325 & 244 \\
\hline Tipo de suelo & S 3 & S 3 & S 3 \\
\hline \multicolumn{4}{|l|}{ Mejor modelo SPAC } \\
\hline Observ & todos los anill & 1 capa 16,5 & comb sin 2 anillos \\
\hline misfit & 0,38 & 0,47 & 0,25 \\
\hline $\mathrm{Vs} 1(\mathrm{~m} / \mathrm{s})$ & 240 & 205 & 244 \\
\hline H1 (m) & 17,46 & 16,51 & 20 \\
\hline Vs2 (m/s) & & & 458 \\
\hline $\mathrm{H} 2(\mathrm{~m})$ & & & 34 \\
\hline \multicolumn{4}{|l|}{$\mathrm{Vs} 3(\mathrm{~m} / \mathrm{s})$} \\
\hline \multicolumn{4}{|l|}{ H3 (m) } \\
\hline Vbasam (m/S) & 700 & 529 & 584 \\
\hline Periodo (s)1 & 0,29 & 0,42 & 0,6 \\
\hline Frecuencia & 3,45 & 2,38 & 1,67 \\
\hline Vs30 (m/s) & 331 & 283 & 289 \\
\hline Tipo de suelo & S 3 & S 3 & S 3 \\
\hline
\end{tabular}


$\mathrm{S}_{2}$ a $\mathrm{S}_{3}$ (en el límite entre ambas clasificaciones): SLPF, ing. eléctrica UCR (2 sitios, 13\%).

$\mathrm{S}_{2}(350 \mathrm{~m} / \mathrm{s}<\mathrm{Vs} 30<760 \mathrm{~m} / \mathrm{s}):$ CTEC, SHTH (2 sitios, 13\%).

$\mathrm{S}_{1}$ a $\mathrm{S}_{2}$ : SJUD (1 sitio, 7\%)

$\mathrm{S}_{1}(\mathrm{Vs} 30>760 \mathrm{~m} / \mathrm{s})$ : SJUD (ningún sitio, 0\%).

En general, se observa que los sitios clasificados según el método de arrays (Vs30) predominan los tipo S3 (según CSCR-2010) y usando $\mathrm{H} / \mathrm{V}$ (identificación de $\mathrm{T}_{0} \mathrm{y}$ aplicando Zhao et al, 2006), predomina S IV. Lo anterior se debe a que los límites de Vs30 definidos por ambos criterios difieren ligeramente, pero en general se está hablando del mismo tipo de suelo. En esta investigación se adopta la clasificación dada por el CSCR-2010, que es la oficial en el país.

En sitios como APMR y CCRT el periodo fundamental obtenido con arrays representa solo una parte del fundamental leído según las razones espectrales $\mathrm{H} / \mathrm{V}$, ya que las limitaciones en las aperturas del array impiden obtener información de capas más profundas.

El sitio en el que se manifiestan inconsistencias sustanciales en las clasificaciones de suelos consideradas es en CTEC, ya que según Zhao et al, (2006) clasifica como S IV y según CSCR-2010 corresponde a $\mathrm{S}_{2}$. Esto se debe a irregularidades en el sitio que deben ser analizadas con más detalle, aplicando técnicas similares en otros sitios cercanos, que permitan evidenciar las variaciones del suelo en distancias cortas.

Los modelos más óptimos obtenidos para cada sitio, se pueden resumir de la manera siguiente:

- De una capa: AFBR, ASMR

- De dos capas: AALA, AGRE, APMR, CCRT, CPAR, SHTH, SSBN, SLPF, ing. eléctrica UCR, ecológico UCR y pl. Iglesias Cartago.

- De cuatro capas: CTEC (además de inversión de Vs en la primera capa)

- Sin modelo: SJUD: suelo homogéneo y sin dispersión.

\section{AGRADECIMIENTOS}

Las siguientes personas tuvieron una significativa participación en este proyecto, especialmente en el trabajo de campo: Esteban Cordero (técnico en electrónica), José Pablo Echeverría (asistente de investigación) Ana Laura Acuña Ortega (investigadora), Albert Macau Roig (IGC, Barcelona, España).

Se contó con el apoyo financiero por parte del Fondo de Incentivos del MICIT-CONICIT, específicamente para intercambio de profesionales entre países y viáticos para las giras dentro del país.

\section{REFERENCIAS BIBLIOGRÁFICAS}

AKI, K., 1957: Space and time spectra of stationary stochastic waves, with special reference to microtremors.- Bull. Earthquake Res. Inst. Tokio, 35: 415-456.

BUILDING SEISMIC SOCIETY COUNCIL (BSSC), 2003: The 2003 NEHRP recommended provisions for new buildings and other structures, Part 1 (Provisions) and Part II (Commentary).- FEMA 368/369, Washington D.C.

\section{COLEGIO FEDERADO DE INGENIEROS Y DE} ARQUITECTOS DE COSTA RICA (CFIA), 2010: Código Sísmico de Costa Rica 2002 [4ta ed.].- 288 págs. Editorial Tecnológica de Costa Rica, Cartago.

KRAMER, S. L., 1996: Geotechnical earthquake engineering.- 653 págs. Prentice-Hall, New Jersey.

NAKAMURA, Y., 1989: A method of dynamic characteristics estimation of subsurface using microtremor on the ground surface.Railways Technical Res. Inst. Quarterly Reports, 30(1): 25-33. 
SESAME EUROPEAN RESEARCH PROJECT, 2004: Guidelines for the implementation of the $\mathrm{H} / \mathrm{V}$ spectral ratio technique on ambient vibration. Measurements, processing and interpretations.- 62 págs. European Commission - Research General Directorate, Project No. EVG1CT-2000-00026 SESAME.

WATHELET, M., 2007: Array recordings of ambient vibrations: surface-wave inversions.- 185 págs. Université Liège, Bélgica [Tesis Ph.D].

ZHAO, J., ASANO, A., FUKOSHIMA, Y., IRIKURA, K., JOHN, X., OGAWA, H., OHNO, Y., OOUCHI, T., SOMERVILLE, P. G., TAKAHASHI, T. \& ZHANG, J., 2006: An empirical siteclassification method for strong-motion in Japan using H/V response spectral ratio.Bull. Sesism. Soc. Am. 96(3): 914-925. 